\title{
Positive Solutions to Fractional Boundary Value Problems with Nonlinear Boundary Conditions
}

\author{
Nemat Nyamoradi, ${ }^{1}$ Dumitru Baleanu, ${ }^{2,3,4}$ and Tahereh Bashiri ${ }^{1}$ \\ ${ }^{1}$ Department of Mathematics, Faculty of Sciences, Razi University, Kermanshah 67149, Iran \\ ${ }^{2}$ Department of Mathematics and Computer Sciences, Faculty of Art and Sciences, Cankaya University, 06530 Ankara, Turkey \\ ${ }^{3}$ Institute of Space Sciences, P.O. BOX MG-23, Magurele, 76900 Bucharest, Romania \\ ${ }^{4}$ Department of Chemical and Materials Engineering, Faculty of Engineering, King Abdulaziz University, P.O. Box 80204, \\ Jeddah 21589, Saudi Arabia
}

Correspondence should be addressed to Nemat Nyamoradi; neamat80@yahoo.com

Received 20 March 2013; Accepted 5 April 2013

Academic Editor: Juan J. Trujillo

Copyright ( 2013 Nemat Nyamoradi et al. This is an open access article distributed under the Creative Commons Attribution License, which permits unrestricted use, distribution, and reproduction in any medium, provided the original work is properly cited.

We consider a system of boundary value problems for fractional differential equation given by $D_{0^{+}}^{\beta} \phi_{p}\left(D_{0^{+}}^{\alpha} u\right)(t)=\lambda_{1} a_{1}(t) f_{1}(u(t)$, $v(t)), t \in(0,1), D_{0^{+}}^{\beta} \phi_{p}\left(D_{0^{+}}^{\alpha} v\right)(t)=\lambda_{2} a_{2}(t) f_{2}(u(t), v(t)), t \in(0,1)$, where $1<\alpha, \beta \leq 2,2<\alpha+\beta \leq 4, \lambda_{1}$, $\lambda_{2}$ are eigenvalues, subject either to the boundary conditions $D_{0^{+}}^{\alpha} u(0)=D_{0^{+}}^{\alpha} u(1)=0, u(0)=0, D_{0^{+}}^{\beta_{1}} u(1)-\sum_{i=1}^{m-2} a_{1 i} D_{0^{+}}^{\beta_{1}} u\left(\xi_{1 i}\right)=0, D_{0^{+}}^{\alpha} v(0)=D_{0^{+}}^{\alpha} v(1)=0$, $v(0)=0, D_{0^{+}}^{\beta_{1}} v(1)-\sum_{i=1}^{m-2} a_{2 i} D_{0^{+}}^{\beta_{1}} v\left(\xi_{2 i}\right)=0$ or $D_{0^{+}}^{\alpha} u(0)=D_{0^{+}}^{\alpha} u(1)=0, u(0)=0, D_{0^{+}}^{\beta_{1}} u(1)-\sum_{i=1}^{m-2} a_{1 i} D_{0^{+}}^{\beta_{1}} u\left(\xi_{1 i}\right)=\psi_{1}(u), D_{0^{+}}^{\alpha} v(0)=$ $D_{0^{+}}^{\alpha} v(1)=0, v(0)=0, D_{0^{+}}^{\beta_{1}} v(1)-\sum_{i=1}^{m-2} a_{2 i} D_{0^{+}}^{\beta_{1}} v\left(\xi_{2 i}\right)=\psi_{2}(v)$, where $0<\beta_{1}<1, \alpha-\beta_{1}-1 \geq 0$ and $\psi_{1}, \psi_{2}: C([0,1]) \rightarrow[0, \infty)$ are continuous functions. The Krasnoselskiis fixed point theorem is applied to prove the existence of at least one positive solution for both fractional boundary value problems. As an application, an example is given to demonstrate some of main results.

\section{Introduction}

Fractional calculus is the field of mathematical analysis which deals with the investigation and applications of integrals and derivatives of arbitrary order; the fractional calculus may be considered an old and yet novel topic.

Recently, fractional differential equations have found numerous applications in various fields of physics and engineering $[1,2]$. It should be noted that most of the books and papers on fractional calculus are devoted to the solvability of initial value problems for differential equations of fractional order. In contrast, the theory of boundary value problems for nonlinear fractional differential equations has received attention quite recently and many aspects of this theory need to be explored. For more details and examples, see [3-9] and the references therein; moreover, fractional derivative arises from many physical processes, such as a charge transport in amorphous semiconductors [10]; electrochemistry and material science are also described by differential equations of fractional order [11-15]. In [16], Bai and Lü considered the boundary value problem of fractional order differential equation

$$
\begin{gathered}
D_{0^{+}}^{\alpha} u(t)+f(t, u(t))=0, \quad t \in(0,1), \\
u(0)=u(1)=0,
\end{gathered}
$$

where $D_{0^{+}}^{\alpha}$ is the standard Riemann-Liouville fractional derivative of order $1<\alpha \leq 2$ and $f:[0,1] \times[0, \infty) \rightarrow[0, \infty)$ is continuous.

In [17], Salem considered the following nonlinear $m$ point boundary value problem of fractional type:

$$
\begin{array}{r}
D_{0^{+}}^{\alpha} x(t)+q(t) f(t, x(t))=0, \quad \text { a.e. on }[0,1], \\
\alpha \in(n-1, n], n \geq 2, \\
x(0)=x^{\prime}(0)=x^{\prime \prime}(0)=\cdots=x^{(n-2)}(0)=0, \\
x(1)=\sum_{i=1}^{m-2} \xi_{i} x\left(\eta_{i}\right),
\end{array}
$$


where $0<\eta_{1}<\cdots<\eta_{m-2}<1, \xi_{i}>0$ with $\sum_{i=1}^{m-2} \xi_{i} \eta_{i}^{\alpha-1}<1$, $q$ is a real valued continuous function, and $f$ is a nonlinear Pettis integrable function.

The turbulent flow in a porous medium is a fundamental mechanics problem. For studying this type of problems, Leibenson [18] introduced the $p$-Laplacian equation as follows:

$$
\left(\phi_{p}\left(x^{\prime}(t)\right)\right)^{\prime}=f\left(t, x(t), x^{\prime}(t)\right)
$$

where $\phi_{p}(s)=|s|^{p-2} s, p>1$. Obviously, $\phi_{p}$ is invertible and its inverse operator is $\phi_{q}$, where $q>1$ is a constant such that $(1 / q)+(1 / p)=1$

Ahmad et al. [19] also considered the existence of solutions for the following three-point boundary value problem of Langevin equation with two different fractional orders:

$$
\begin{gathered}
{ }^{c} D^{\beta}\left({ }^{c} D^{\beta}+\lambda\right) x(t)=f(t, x(t)), \\
0<t<1,1<\beta \leq 2,0<\alpha \leq 1, \\
x(0)=0, \quad x(\eta)=0, \quad x(1)=0, \quad 0<\eta<1,
\end{gathered}
$$

where ${ }^{c} D$ is the Caputo fractional derivative, $f:[0,1] \times \mathbb{R} \rightarrow$ $\mathbb{R}$ is a given continuous function, and $\lambda$ is a real number.

Dai [20] considered the following problem of ordinary differential equations:

$$
\begin{gathered}
\left(\phi_{p}\left(u^{\prime}\right)\right)^{\prime}=\lambda a(t) f(u), \quad t \in(0,1), \\
u(0)=u(1)=0 .
\end{gathered}
$$

By means of global bifurcation techniques and the approximation of connected components, existence and multiplicity results for positive solutions were obtained.

Motivated by the works above, our purpose in this paper is to show the existence of at least one positive solution for the following fractional $p$-Laplacian system:

$$
\begin{array}{ll}
D_{0^{+}}^{\beta} \phi_{p}\left(D_{0^{+}}^{\alpha} u\right)(t)=\lambda_{1} a_{1}(t) f_{1}(u(t), v(t)), & t \in(0,1), \\
D_{0^{+}}^{\beta} \phi_{p}\left(D_{0^{+}}^{\alpha} v\right)(t)=\lambda_{2} a_{2}(t) f_{2}(u(t), v(t)), & t \in(0,1),
\end{array}
$$

where $1<\alpha, \beta \leq 2,2<\alpha+\beta \leq 4, D_{0^{+}}^{\alpha}$ is the RiemannLiouville fractional derivative of order $\alpha, \lambda_{1}, \lambda_{2}>0$, and $m>$ 2 is integer.

We first consider the problem (6) with following boundary condition:

$$
\begin{gathered}
D_{0^{+}}^{\alpha} u(0)=D_{0^{+}}^{\alpha} u(1)=0, \quad u(0)=0, \\
D_{0^{+}}^{\beta_{1}} u(1)-\sum_{i=1}^{m-2} a_{1 i} D_{0^{+}}^{\beta_{1}} u\left(\xi_{1 i}\right)=0, \\
D_{0^{+}}^{\alpha} v(0)=D_{0^{+}}^{\alpha} v(1)=0, \quad v(0)=0, \\
D_{0^{+}}^{\beta_{1}} v(1)-\sum_{i=1}^{m-2} a_{2 i} D_{0^{+}}^{\beta_{1}} v\left(\xi_{2 i}\right)=0 .
\end{gathered}
$$

We then consider the case in which the boundary conditions are changed to

$$
\begin{gathered}
D_{0^{+}}^{\alpha} u(0)=D_{0^{+}}^{\alpha} u(1)=0, \quad u(0)=0, \\
D_{0^{+}}^{\beta_{1}} u(1)-\sum_{i=1}^{m-2} a_{1 i} D_{0^{+}}^{\beta_{1}} u\left(\xi_{1 i}\right)=\psi_{1}(u), \\
D_{0^{+}}^{\alpha} v(0)=D_{0^{+}}^{\alpha} v(1)=0, \quad v(0)=0, \\
D_{0^{+}}^{\beta_{1}} v(1)-\sum_{i=1}^{m-2} a_{2 i} D_{0^{+}}^{\beta_{1}} v\left(\xi_{2 i}\right)=\psi_{2}(v),
\end{gathered}
$$

where $\psi_{1}, \psi_{2}: C([0,1]) \rightarrow[0, \infty)$ are continuous functions, where $C([0,1])$ means the set of continuous, real valued functions on the unit interval $[0,1]$.

In the cases, we assume that $0<\beta_{1}<1, \alpha-\beta_{1}-1 \geq 0$.

In the past few decades, many important results relative to (6) with certain boundary value conditions have been obtained; we refer the reader to [21-25] and the references therein.

The following conditions will be used in this paper:

(H1) $\phi_{p}(s)=|s|^{p-2} s, p>1$ is a $p$-laplacian operator. Obviously, $\phi_{p}$ is invertible and $\phi_{p}^{-1}=\phi_{q}$, where $q>1$ is a constant such that $(1 / q)+(1 / p)=1$;

(H2) $0<\xi_{j 1}<\xi_{j 2}<\cdots<\xi_{j m-2}<1, a_{j i}>0$ for $i=$ $1,2, \ldots, m-2$ and $\sum_{i=1}^{m-2} a_{j i} \xi_{j i}^{\alpha-\beta_{1}-1}<1, j=1,2 ;$

(H3) $f_{j}:[0,+\infty) \times[0,+\infty) \rightarrow[0,+\infty)$ is a given continuous function and $a_{j}$ is a positive real valued continuous function, $j=1,2$.

The rest of the paper is organized as follows: in Section 2, we will recall certain results from the theory of the continuous fractional calculus; in Section 3, we will provide some conditions under which the problem (6) and (7) has at least one positive solution; in Section 4, by suitable conditions, we will prove that the problem (6) and (8) has at least one positive solution; finally, in Section 4, we will provide some numerical examples, which will explicate the applicability of our results.

\section{Preliminaries}

In this section, we present some notations and preliminary lemmas that will be used in the proofs of the main results.

Definition 1. Let $X$ be a real Banach space. A nonempty closed set $P \subset X$ is called a cone of $X$ if it satisfies the following conditions:

(1) $x \in P, \mu \geq 0$, implies $\mu x \in P$,

(2) $x \in P,-x \in P$, implies $x=0$.

Definition 2 (see $[26,27])$. The Riemann-Liouville fractional integral operator of order $\alpha>0$ of function $f \in L^{1}\left(\mathbb{R}^{+}\right)$is defined as

$$
I_{0^{+}}^{\alpha} f(t)=\frac{1}{\Gamma(\alpha)} \int_{0}^{t}(t-s)^{\alpha-1} f(s) d s,
$$

where $\Gamma(\cdot)$ is the Euler gamma function. 
Definition 3 (see $[26,27]$ ). The Riemann-Liouville fractional derivative of order $\alpha>0$ of a continuous function $f:(0, \infty) \rightarrow \mathbb{R}$ is defined as

$$
D_{0^{+}}^{\alpha} f(t)=\frac{1}{\Gamma(n-\alpha)}\left(\frac{d}{d t}\right)^{n} \int_{0}^{t}(t-s)^{n-\alpha-1} f(s) d s,
$$

where $n=[\alpha]+1$.

Lemma 4 (see [28]). The equality $D_{0^{+}}^{\gamma} I_{0^{+}}^{\gamma} f(t)=f(t), \gamma>0$, holds for $f \in L^{1}(0,1)$.

Lemma 5 (see [28]). Let $\alpha>0$. Then the differential equation

$$
D_{0^{+}}^{\alpha} u=0
$$

has a unique solution $u(t)=c_{1} t^{\alpha-1}+c_{2} t^{\alpha-2}+\cdots+c_{n} t^{\alpha-n}, c_{i} \in \mathbb{R}$, $i=1, \ldots, n$, where $n-1<\alpha \leq n$.

Lemma 6 (see [28]). Let $\alpha>0$. Then the following equality holds for $u \in L^{1}(0,1), D_{0^{+}}^{\alpha} u \in L^{1}(0,1)$,

$$
I_{0^{+}}^{\alpha} D_{0^{+}}^{\alpha} u(t)=u(t)+c_{1} t^{\alpha-1}+c_{2} t^{\alpha-2}+\cdots+c_{n} t^{\alpha-n},
$$

$c_{i} \in \mathbb{R}, i=1, \ldots, n$, where $n-1<\alpha \leq n$.

In the following, we present the Green function of fractional differential equation boundary value problem.

Let

$$
y(t)=-\phi_{p}\left(D_{0^{+}}^{\alpha} u\right)(t)
$$

then, the problem

$$
\begin{gathered}
D_{0^{+}}^{\beta} \phi_{p}\left(D_{0^{+}}^{\alpha} u\right)(t)=h(t), \quad 1<\beta \leq 2, t \in(0,1), \\
D_{0^{+}}^{\alpha} u(0)=D_{0^{+}}^{\alpha} u(1)=0,
\end{gathered}
$$

where $h \in C[0,1]$, is turned into problem

$$
\begin{gathered}
D_{0^{+}}^{\beta} y(t)+h(t)=0, \quad 1<\beta \leq 2, t \in(0,1), \\
y(0)=y(1)=0 .
\end{gathered}
$$

Lemma 7. Suppose that $h \in C[0,1]$, then the boundary value problem (15) has a unique solution

$$
y(t)=\int_{0}^{1} H(t, s) h(s) d s
$$

where

$$
\begin{aligned}
& H(t, s) \\
& = \begin{cases}\frac{t^{\beta-1}(1-s)^{\beta-1}-(t-s)^{\beta-1}}{\Gamma(\beta)}, & 0 \leq s \leq t \leq 1, \\
\frac{t^{\beta-1}(1-s)^{\beta-1}}{\Gamma(\beta)}, & 0 \leq t \leq s \leq 1 .\end{cases}
\end{aligned}
$$

Proof. The proof is similar to that of Lemma 2.3 in [16], so we omit it here.
Lemma 8 (see [16]). For $\lambda>-1$ and $\alpha>0$,

$$
D_{0^{+}}^{\alpha} t^{\gamma}=\frac{\Gamma(\gamma+1)}{\Gamma(\gamma-\alpha+1)} t^{\gamma^{-\alpha}} \text {. }
$$

Lemma 9 (see [29]). Suppose that $g \in L^{1}(0,1)$ and $\alpha, \beta$ are two constants such that $0 \leq \beta \leq 1<\alpha$; then,

$$
D_{0^{+}}^{\beta} \int_{0}^{t}(t-s)^{\alpha-1} g(s) d s=\frac{\Gamma(\alpha)}{\Gamma(\alpha-\beta)} \int_{0}^{t}(t-s)^{\alpha-\beta-1} g(s) d s .
$$

Lemma 10. Suppose that (H1) and (H2) hold. Then, for $y \in$ $C[0,1]$, the boundary value problem

$$
\begin{array}{r}
D_{0^{+}}^{\alpha} u(t)+\phi_{q}(y(t))=0, \quad t \in(0,1), 1<\alpha \leq 2, \\
u(0)=0, \quad D_{0^{+}}^{\beta_{1}} u(1)-\sum_{i=1}^{m-2} a_{j i} D_{0^{+}}^{\beta_{1}} u\left(\xi_{j i}\right)=0, \\
0<\beta_{1}<1,
\end{array}
$$

has a unique solution

$$
\begin{aligned}
u(t)= & \int_{0}^{1} G(t, s) \phi_{q}(y(s)) d s \\
& +\frac{t^{\alpha-1}}{\left(1-\Delta_{j}\right)} \sum_{i=1}^{m-2} a_{j i} \int_{0}^{1} G_{1}\left(\xi_{j i}, s\right) \phi_{q}(y(s)) d s,
\end{aligned}
$$

where $\Delta_{j}=\sum_{i=1}^{m-2} a_{j i} \xi_{j i}^{\alpha-\beta_{1}-1} \neq 1$, for $j=1,2$,

$G(t, s)$

$$
= \begin{cases}\frac{t^{\alpha-1}(1-s)^{\alpha-\beta_{1}-1}-(t-s)^{\alpha-1}}{\Gamma(\alpha)}, & 0 \leq s \leq t \leq 1, \\ \frac{t^{\alpha-1}(1-s)^{\alpha-\beta_{1}-1}}{\Gamma(\alpha)}, & 0 \leq t \leq s \leq 1,\end{cases}
$$

$G_{1}(t, s)$

$$
= \begin{cases}\frac{t^{\alpha-\beta_{1}-1}(1-s)^{\alpha-\beta_{1}-1}-(t-s)^{\alpha-\beta_{1}-1}}{\Gamma(\alpha)}, & 0 \leq s \leq t \leq 1, \\ \frac{t^{\alpha-\beta_{1}-1}(1-s)^{\alpha-\beta_{1}-1}}{\Gamma(\alpha)}, & 0 \leq t \leq s \leq 1 .\end{cases}
$$

Proof. By applying Lemma 6, (20) is equivalent to the following integral equation:

$$
u(t)=-\frac{1}{\Gamma(\alpha)} \int_{0}^{t}(t-s)^{\alpha-1} \phi_{q}(y(s)) d s-c_{1} t^{\alpha-1}-c_{2} t^{\alpha-2},
$$

for some arbitrary constants $c_{1}, c_{2} \in \mathbb{R}$.

By the boundary condition $u(0)=0$, we conclude that $c_{2}=0$; then we have

$$
u(t)=-\frac{1}{\Gamma(\alpha)} \int_{0}^{t}(t-s)^{\alpha-1} \phi_{q}(y(s)) d s-c_{1} t^{\alpha-1}
$$


It follows from Lemmas 8 and 9 that

$$
\begin{aligned}
D_{0^{+}}^{\beta_{1}} u(t)= & -\frac{1}{\Gamma\left(\alpha-\beta_{1}\right)} \\
& \times \int_{0}^{t}(t-s)^{\alpha-\beta_{1}-1} \phi_{q}(y(s)) d s \\
& -c_{1} \frac{\Gamma(\alpha)}{\Gamma\left(\alpha-\beta_{1}\right)} t^{\alpha-\beta_{1}-1} .
\end{aligned}
$$

So, by the boundary condition $D_{0^{+}}^{\beta_{1}} u(1)-\sum_{i=1}^{m-2} a_{j i} D_{0^{+}}^{\beta_{1}} u\left(\xi_{j i}\right)=$ 0 , we obtain that

$$
\begin{aligned}
c_{1}= & -\frac{1}{\Gamma(\alpha)\left(1-\Delta_{j}\right)} \int_{0}^{1}(1-s)^{\alpha-\beta_{1}-1} \phi_{q}(y(s)) d s \\
& +\frac{1}{\Gamma(\alpha)\left(1-\Delta_{j}\right)} \sum_{i=1}^{m-2} a_{j i} \\
& \times \int_{0}^{\xi_{j i}}\left(\xi_{j i}-s\right)^{\alpha-\beta_{1}-1} \phi_{q}(y(s)) d s .
\end{aligned}
$$

Then, the unique solution of (20) is given by the formula

$$
\begin{aligned}
u(t)= & -\frac{1}{\Gamma(\alpha)} \int_{0}^{t}(t-s)^{\alpha-1} \phi_{q}(y(s)) d s \\
& +\frac{1}{\left(1-\Delta_{j}\right) \Gamma(\alpha)} \int_{0}^{1} t^{\alpha-1}(1-s)^{\alpha-\beta_{1}-1} \phi_{q}(y(s)) d s \\
& -\frac{t^{\alpha-1}}{\left(1-\Delta_{j}\right) \Gamma(\alpha)} \\
& \times \sum_{i=1}^{m-2} a_{j i} \int_{0}^{\xi_{j i}}\left(\xi_{j i}-s\right)^{\alpha-\beta_{1}-1} \phi_{q}(y(s)) d s \\
= & -\frac{1}{\Gamma(\alpha)} \int_{0}^{t}(t-s)^{\alpha-1} \phi_{q}(y(s)) d s \\
& +\frac{1}{\Gamma(\alpha)} \int_{0}^{1} t^{\alpha-1}(1-s)^{\alpha-\beta_{1}-1} \phi_{q}(y(s)) d s \\
& +\frac{\Delta_{j}}{\left(1-\Delta_{j}\right) \Gamma(\alpha)} \int_{0}^{1} t^{\alpha-1}(1-s)^{\alpha-\beta_{1}-1} \phi_{q}(y(s)) d s \\
& +\frac{\sum^{\alpha-1}}{\left(1-\Delta_{j}\right) \Gamma(\alpha)} \\
& \sum_{j=1}^{\xi_{j i}}\left(\xi_{j i}-s\right)^{\alpha-\beta_{1}-1} \phi_{q}(y(s)) d s
\end{aligned}
$$

$$
\begin{aligned}
& =\frac{1}{\Gamma(\alpha)}\left[\int_{0}^{t}\left[t^{\alpha-1}(1-s)^{\alpha-\beta_{1}-1}-(t-s)^{\alpha-1}\right]\right. \\
& \times \phi_{q}(y(s)) d s \\
& \left.+\int_{t}^{1} t^{\alpha-1}(1-s)^{\alpha-\beta_{1}-1} \phi_{q}(y(s)) d s\right] \\
& +\frac{t^{\alpha-1}}{\left(1-\Delta_{j}\right) \Gamma(\alpha)} \\
& \times \sum_{i=1}^{m-2} a_{j i} \int_{0}^{1} \xi_{j i}^{\alpha-\beta_{1}-1}(1-s)^{\alpha-\beta_{1}-1} \phi_{q}(y(s)) d s \\
& -\frac{t^{\alpha-1}}{\left(1-\Delta_{j}\right) \Gamma(\alpha)} \\
& \times \sum_{i=1}^{m-2} a_{j i} \int_{0}^{\xi_{j i}}\left(\xi_{j i}-s\right)^{\alpha-\beta_{1}-1} \phi_{q}(y(s)) d s \\
& =\frac{1}{\Gamma(\alpha)}\left[\int_{0}^{t}\left[t^{\alpha-1}(1-s)^{\alpha-\beta_{1}-1}-(t-s)^{\alpha-1}\right]\right. \\
& \times \phi_{q}(y(s)) d s \\
& \left.+\int_{t}^{1} t^{\alpha-1}(1-s)^{\alpha-\beta-1} \phi_{q}(y(s)) d s\right] \\
& +\frac{t^{\alpha-1}}{\left(1-\Delta_{j}\right) \Gamma(\alpha)} \\
& \times \sum_{i=1}^{m-2} a_{j i}\left[\int _ { 0 } ^ { \xi _ { j i } } \left[(1-s)^{\alpha-\beta-1} \xi_{j i}^{\alpha-\beta_{1}-1}\right.\right. \\
& \left.-\left(\xi_{j i}-s\right)^{\alpha-\beta_{1}-1}\right] \phi_{q}(y(s)) d s \\
& +\int_{\xi_{j i}}^{1}(1-s)^{\alpha-\beta_{1}-1} \\
& \left.\times \xi_{j i}^{\alpha-\beta_{1}-1} \phi_{q}(y(s)) d s\right] \\
& =\int_{0}^{1} G(t, s) \phi_{q}(y(s)) d s \\
& +\frac{t^{\alpha-1}}{\left(1-\Delta_{j}\right)} \sum_{i=1}^{m-2} a_{j i} \int_{0}^{1} G_{1}\left(\xi_{j i}, s\right) \phi_{q}(y(s)) d s .
\end{aligned}
$$

Then, the proof is completed.

Lemma 11. Assume $\alpha-\beta_{1}-1 \geq 0$, then; for all $(t, s) \in[0,1] \times$ $[0,1]$, we have

(i) $0 \leq G_{1}(t, s) \leq(1 / \Gamma(\alpha)) t^{\alpha-\beta_{1}-1}(1-s)^{\alpha-\beta_{1}-1}, 0 \leq$ $G(t, s) \leq G(s, s)$, for any $t, s \in[0,1]$;

(ii) there exists a positive function $g \in C[0,1]$ such that $\min _{\gamma \leq t \leq \delta} G(t, s) \geq g(s) G(s, s), s \in(0,1)$, 
where

$$
g(s)= \begin{cases}\frac{\delta^{\alpha-1}(1-s)^{\alpha-\beta_{1}-1}-(\delta-s)^{\alpha-1}}{s^{\alpha-1}(1-s)^{\alpha-\beta_{1}-1}}, & s \in(0, r], \\ \left(\frac{\gamma}{s}\right)^{\alpha-1}, & s \in[r, 1),\end{cases}
$$

with $\gamma<r<\delta$.

Proof. (i) If $0 \leq s \leq t \leq 1$, we have

$$
\begin{aligned}
G_{1}(t, s) & =\frac{1}{\Gamma(\alpha)} t^{\alpha-\beta_{1}-1}(1-s)^{\alpha-\beta_{1}-1}-(t-s)^{\alpha-\beta_{1}-1} \\
& \geq \frac{1}{\Gamma(\alpha)} t^{\alpha-\beta_{1}-1}(1-s)^{\alpha-\beta_{1}-1}-(t-t s)^{\alpha-\beta_{1}-1} \\
& =\frac{1}{\Gamma(\alpha)} t^{\alpha-\beta_{1}-1}\left((1-s)^{\alpha-\beta_{1}-1}-(1-s)^{\alpha-\beta_{1}-1}\right)=0 .
\end{aligned}
$$

If $0 \leq t \leq s \leq 1$, we get

$$
G_{1}(t, s)=\frac{1}{\Gamma(\alpha)} t^{\alpha-\beta_{1}-1}(1-s)^{\alpha-\beta_{1}-1} \geq 0
$$

Thus, $G_{1}(t, s) \geq 0$, for any $(t, s) \in[0,1] \times[0,1]$. It is obvious that $G_{1}(t, s) \leq(1 / \Gamma(\alpha)) t^{\alpha-\beta_{1}-1}(1-s)^{\alpha-\beta_{1}-1}$.

Now, we show that $0 \leq G(t, s) \leq G(s, s)$ for any $t, s \in$ $[0,1]$. We define

$$
\begin{gathered}
g_{1}(t, s)=\frac{t^{\alpha-1}(1-s)^{\alpha-\beta_{1}-1}-(t-s)^{\alpha-1}}{\Gamma(\alpha)}, \\
0 \leq s \leq t \leq 1, \\
g_{2}(t, s)=\frac{t^{\alpha-1}(1-s)^{\alpha-\beta_{1}-1}}{\Gamma(\alpha)}, \\
0 \leq t \leq s \leq 1 .
\end{gathered}
$$

One can get

$$
\begin{aligned}
g_{1}(t, s) & \geq \frac{1}{\Gamma(\alpha)}\left[t^{\alpha-1}(1-s)^{\alpha-\beta_{1}-1}-(t-t s)^{\alpha-1}\right] \\
& =\frac{1}{\Gamma(\alpha)}\left[t^{\alpha-1}\left((1-s)^{\alpha-\beta_{1}-1}-(1-s)^{\alpha-1}\right)\right] \geq 0,
\end{aligned}
$$

on the other hand, it is obvious that $g_{2}(t, s) \geq 0,0 \leq t \leq s \leq 1$.

Thus

$$
G(t, s) \geq 0, \quad \forall(t, s) \in[0,1] \times[0,1] .
$$

For any $0 \leq s \leq t \leq 1$,

$$
\begin{aligned}
\frac{\partial g_{1}(t, s)}{\partial t}= & \frac{1}{\Gamma(\alpha)}\left[(\alpha-1) t^{\alpha-2}(1-s)^{\alpha-\beta_{1}-1}\right. \\
& \left.-(\alpha-1)(t-s)^{\alpha-2}\right] \\
= & \frac{1}{\Gamma(\alpha)}(\alpha-1) t^{\alpha-2} \\
& \times\left[(1-s)^{\alpha-\beta_{1}-1}-\left(1-\frac{s}{t}\right)^{\alpha-2}\right] \\
\leq & \frac{1}{\Gamma(\alpha)}(\alpha-1) t^{\alpha-2} \\
& \times\left[(1-s)^{\alpha-\beta_{1}-1}-(1-s)^{\alpha-2}\right] \\
\leq & 0,
\end{aligned}
$$

then, $g_{1}(t, s)$ is nonincreasing with respect to $t$ on $[s, 1]$; hence, we obtain that

$$
g_{1}(t, s) \leq g_{1}(s, s), \quad \forall 0 \leq s \leq t \leq 1 .
$$

Also, we have

$$
\frac{\partial g_{2}(t, s)}{\partial t}=\frac{1}{\Gamma(\alpha)}\left[(\alpha-1) t^{\alpha-2}(1-s)^{\alpha-\beta_{1}-1}\right] \geq 0,
$$

then, $g_{2}(t, s)$ is increasing with respect to $t$ on $[0, s]$. Then, by the fact that $g_{1}(s, s)=g_{2}(s, s)$, we have

$$
G(t, s) \leq G(s, s), \quad \forall t, s \in[0,1] .
$$

(ii) Since $g_{1}(\cdot, s)$ is nonincreasing and $g_{2}(\cdot, s)$ is nondecreasing, for all $s, t \in[0,1]$, we have

$$
\begin{aligned}
\min _{\gamma \leq t \leq \delta} G(t, s) & = \begin{cases}\min _{\gamma \leq t \leq \delta} g_{1}(t, s), & s \in[0, \gamma], \\
\min _{\gamma \leq t \leq \delta}\left\{g_{1}(t, s), g_{2}(t, s)\right\}, & s \in[\gamma, \delta], \\
\min _{\gamma \leq t \leq \delta} g_{2}(t, s), & s \in[\delta, 1],\end{cases} \\
& = \begin{cases}\min _{\gamma \leq t \leq \delta} g_{1}(t, s), & s \in[0, r], \\
\min _{\gamma \leq t \leq \delta} g_{2}(t, s), & s \in[r, 1],\end{cases} \\
\geq \begin{cases}g_{1}(\delta, s), & s \in[0, r], \\
g_{2}(\gamma, s), & s \in[r, 1],\end{cases} & \begin{cases}\delta^{\alpha-1}(1-s)^{\alpha-\beta_{1}-1}-(\delta-s)^{\alpha-1}, & s \in[0, r], \\
\gamma^{\alpha-1}(1-s)^{\alpha-\beta_{1}-1}, & s, 1],\end{cases}
\end{aligned}
$$

where $\gamma<r<\delta$ is the solution of

$$
\delta^{\alpha-1}(1-r)^{\alpha-\beta_{1}-1}-(\delta-r)^{\alpha-1}=\gamma^{\alpha-1}(1-r)^{\alpha-\beta_{1}-1},
$$

so, we get

$$
\min _{\gamma \leq t \leq \delta} G(t, s) \geq g(s) G(s, s), \quad \forall s \in[0,1],
$$

where $g(s)$ is given in (28). This completes the proof. 
Remark 12. If $\gamma \in(0,1 / 4)$ and $\delta=1-\gamma$, then Lemma 11 satisfies.

In this paper, we assume that $\gamma \in(0,1 / 4)$ and $\delta=1-\gamma$.

Now, we consider system (6). Assume that (H1), (H2), and (H3) hold; then, by applying Lemmas 7 and 10, $(u, v) \in$ $C(0,1) \times C(0,1)$ is a solution of system (6) if and only if $(u, v) \in C[0,1] \times C[0,1]$ is a solution of the following nonlinear integral system:

$$
\begin{aligned}
u(t)= & \int_{0}^{1} G(t, s) \phi_{q} \\
& \times\left(\lambda_{1} \int_{0}^{1} H(s, r) a_{1}(r) f_{1}(u(r), v(r)) d r\right) d s \\
& +\frac{t^{\alpha-1}}{\left(1-\Delta_{1}\right)} \sum_{i=1}^{m-2} a_{1 i} \\
& \times \int_{0}^{1} G_{1}\left(\xi_{1 i}, s\right) \phi_{q} \\
& \times\left(\lambda_{1} \int_{0}^{1} H(s, r) a_{1}(r)\right. \\
v(t)= & \int_{0}^{1} G(t, s) \phi_{q} \\
& \times\left(\lambda_{2} \int_{0}^{1} H(s, r) a_{2}(r) f_{2}(u(r), v(r)) d r\right) d s \\
& +\frac{t^{\alpha-1}}{\left(1-\Delta_{2}\right)} \sum_{i=1}^{m-2} a_{2 i} \\
& \times \int_{0}^{1} G_{1}\left(\xi_{2 i}, s\right) \phi_{q} \\
& \times\left(\lambda_{2} \int_{0}^{1} H(s, r) a_{2}(r)\right. \\
& \left.\times f_{2}(u(r), v(r)) d r\right) d s .
\end{aligned}
$$

We next recall the Krasnoselskii's fixed point theorem (see [30]). This lemma will be of use in Sections 3 and 4 of this paper.

Theorem 13. Let $E$ be a Banach space and let $K \subseteq E$ be a cone. Assume that $\Omega_{1}$ and $\Omega_{2}$ are open sets contained in $E$ such that $0 \in \Omega_{1}$ and $\bar{\Omega}_{1} \subseteq \Omega_{2}$. Assume, further, that $T: K \cap\left(\bar{\Omega}_{2} \backslash \Omega_{1}\right) \rightarrow$ $K$ is a completely continuous operator. If eigher

(1) $\|T y\| \leq\|y\|$ for $y \in K \cap \partial \Omega_{1}$ and $\|T y\| \geq\|y\|$ for $y \in K \cap \partial \Omega_{2}$ or

(2) $\|T y\| \geq\|y\|$ for $y \in K \cap \partial \Omega_{1}$ and $\|T y\| \leq\|y\|$ for $y \in K \cap \partial \Omega_{2}$,

then, $T$ has at least one fixed point in $K \cap\left(\bar{\Omega}_{2} \backslash \Omega_{1}\right)$.

\section{Existence of a Positive Solution: Case I}

Let $\gamma \in(0,1 / 4)$ and $\delta=1-\gamma$, and define

$$
\begin{aligned}
\eta & =\min _{\gamma \leq t \leq \delta}\{g(t)\}, \\
\sigma & =\min \left\{\eta, \gamma^{\alpha-1}\right\} .
\end{aligned}
$$

In this section, we consider the following assumption:

(L1) There exist numbers $f_{1}^{*}$ and $f_{2}^{*}$, with $f_{1}^{*}, f_{2}^{*} \in$ $(0,+\infty)$, such that

$$
\begin{array}{r}
\lim _{(u, v) \rightarrow\left(0^{+}, 0^{+}\right)} \frac{f_{1}(u, v)}{\phi_{p}(u+v)}=f_{1}^{*}, \\
\lim _{(u, v) \rightarrow\left(0^{+}, 0^{+}\right)} \frac{f_{2}(u, v)}{\phi_{p}(u+v)}=f_{2}^{*} .
\end{array}
$$

(L2) There exist numbers $f_{1}^{* *}$ and $f_{2}^{* *}$, with $f_{1}^{* *}, f_{2}^{* *} \in$ $(0,+\infty)$, such that

$$
\begin{aligned}
\lim _{(u, v) \rightarrow(\infty, \infty)} \frac{f_{1}(u, v)}{\phi_{p}(u+v)} & =f_{1}^{* *}, \\
\lim _{(u, v) \rightarrow(\infty, \infty)} \frac{f_{2}(u, v)}{\phi_{p}(u+v)} & =f_{2}^{* *} .
\end{aligned}
$$

(L3) There are numbers $M_{j}$ and $m_{j}$, where

$$
\begin{aligned}
M_{j}=\frac{1}{2}[ & \int_{0}^{1} G(s, s) \phi_{q}\left(\int_{0}^{1} H(s, r) a_{j}(r)\left(f_{j}^{*}\right) d r\right) d s \\
& +\frac{1}{\left(1-\Delta_{j}\right)} \sum_{i=1}^{m-2} a_{j i} \\
& \times \int_{0}^{1} G_{1}\left(\xi_{j i}, s\right) \phi_{q} \\
m_{j}=\frac{3}{2 \sigma} & {\left[\int_{0}^{1} G(s, s) \phi_{q}\left(\int_{\gamma}^{\delta} H(s, r) a_{j}(r)\left(f_{j}^{* *}\right) d r\right) d s\right.} \\
& +\frac{1}{\left(1-\Delta_{j}\right)} \sum_{i=1}^{m-2} a_{j i} \\
& \times \int_{0}^{1} G_{1}\left(\xi_{j i}, s\right) \phi_{q} \\
& \left.\times\left(\int_{\gamma}^{\delta} H(s, r) a_{j}(r)\left(f_{j}^{* *}\right) d r\right) d s\right]^{-1},
\end{aligned}
$$

such that $\phi_{p}\left(m_{j}\right)<\lambda_{j}<\phi_{p}\left(M_{j}\right), j=1,2$.

The basic space used in this paper is a real Banach space $E=C([0,1], \mathbb{R}) \times C([0,1], \mathbb{R})$ with the norm $\|(u, v)\|:=\|u\|+$ $\|v\|$, where $\|u\|=\max _{t \in[0,1]}|u(t)|$. 
Then, choose a cone $K \subset E$, by

$$
\begin{array}{r}
K=\{(u, v) \in E \mid u(t) \geq 0, v(t) \geq 0, \\
\left.\min _{\gamma \leq t \leq \delta}(u(t)+v(t)) \geq \frac{\sigma}{3}\|(u, v)\|\right\},
\end{array}
$$

and define an operator $T: E \rightarrow E$ by

$$
T(u, v)(t)=\left(T_{1}(u, v)(t), T_{2}(u, v)(t)\right), \quad \forall t \in(0,1)
$$

where

$$
\begin{aligned}
& T_{1}(u, v)(t)=\int_{0}^{1} G(t, s) \phi_{q} \\
& \times\left(\lambda_{1} \int_{0}^{1} H(s, r) a_{1}(r)\right. \\
& \left.\times f_{1}(u(r), v(r)) d r\right) d s \\
& +\frac{t^{\alpha-1}}{\left(1-\Delta_{1}\right)} \sum_{i=1}^{m-2} a_{1 i} \\
& \times \int_{0}^{1} G_{1}\left(\xi_{1 i}, s\right) \phi_{q} \\
& \times\left(\lambda_{1} \int_{0}^{1} H(s, r) a_{1}(r)\right. \\
& \left.\times f_{1}(u(r), v(r)) d r\right) d s, \\
& T_{2}(u, v)(t)=\int_{0}^{1} G(t, s) \phi_{q} \\
& \times\left(\lambda_{2} \int_{0}^{1} H(s, r) a_{2}(r)\right. \\
& \left.\times f_{2}(u(r), v(r)) d r\right) d s \\
& +\frac{t^{\alpha-1}}{\left(1-\Delta_{2}\right)} \sum_{i=1}^{m-2} a_{2 i} \\
& \times \int_{0}^{1} G_{1}\left(\xi_{2 i}, s\right) \phi_{q} \\
& \times\left(\lambda_{2} \int_{0}^{1} H(s, r) a_{2}(r)\right. \\
& \left.\times f_{2}(u(r), v(r)) d r\right) d s
\end{aligned}
$$

Lemma 14. Suppose that (H1), (H2), and (H3) hold. Then, the operator $T: K \rightarrow K$ is well defined, that is, $T(K) \subseteq K$.
Proof. For any $(u, v) \in K$, by (H1), (H2), (H3) and Lemma 11, $T_{1}(u, v)(t) \geq 0, T_{2}(u, v)(t) \geq 0, t \in[0,1]$, and it follows from (47) that

$$
\begin{array}{rl}
\left\|T_{1}(u, v)\right\|=\int_{0}^{1} & G(s, s) \phi_{q} \\
& \times\left(\lambda_{1} \int_{0}^{1} H(s, r) a_{1}(r)\right. \\
& \left.\times f_{1}(u(r), v(r)) d r\right) d s
\end{array}
$$

$$
\begin{aligned}
& +\frac{1}{\left(1-\Delta_{1}\right)} \sum_{i=1}^{m-2} a_{1 i} \\
& \times \int_{0}^{1} G_{1}\left(\xi_{1 i}, s\right) \phi_{q} \\
& \quad \times\left(\lambda_{1} \int_{0}^{1} H(s, r) a_{1}(r)\right.
\end{aligned}
$$$$
\left.\times f_{1}(u(r), v(r)) d r\right) d s
$$$$
\begin{array}{r}
=\left(\int_{0}^{\gamma}+\int_{\gamma}^{\delta}+\int_{\delta}^{1}\right) \\
\times\left(G(s, s) \phi_{q}\right.
\end{array}
$$$$
\left.\times\left(\int_{0}^{1} H(s, r) f_{1}(u(r), v(r)) d r\right)\right) d s
$$$$
+\frac{1}{\left(1-\Delta_{1}\right)} \sum_{i=1}^{m-2} a_{1 i}
$$$$
\times\left(\int_{0}^{\gamma}+\int_{\gamma}^{\delta}+\int_{\delta}^{1}\right)
$$$$
\times\left(G_{1}\left(\xi_{1 i}, s\right) \phi_{q}\right.
$$$$
\times\left(\lambda_{1} \int_{0}^{1} H(s, r) a_{1}(r)\right.
$$$$
\left.\left.\times f_{1}(u(r), v(r)) d r\right)\right) d s
$$$$
\leq 3\left[\int _ { \gamma } ^ { \delta } G ( s , s ) \phi _ { q } \left(\lambda_{1} \int_{0}^{1} H(s, r) a_{1}(r)\right.\right.
$$$$
\left.\times f_{1}(u(r), v(r)) d r\right) d s
$$

$$
\begin{aligned}
& +\frac{1}{\left(1-\Delta_{1}\right)} \sum_{i=1}^{m-2} a_{1 i} \\
& \times \int_{\gamma}^{\delta} G_{1}\left(\xi_{1 i}, s\right) \phi_{q}
\end{aligned}
$$




$$
\begin{aligned}
& \times\left(\lambda_{1} \int_{0}^{1} H(s, r) a_{1}(r)\right. \\
& \left.\left.\quad \times f_{1}(u(r), v(r)) d r\right) d s\right] .
\end{aligned}
$$

Thus, for any $(u, v) \in K$, it follows from Lemma 11 and (49) that

$$
\begin{aligned}
& \min _{\gamma \leq t \leq \delta} T_{1}(u, v)(t) \\
& =\min _{\gamma \leq t \leq \delta}\left\{\int_{0}^{1} G(t, s) \phi_{q}\right. \\
& \times\left(\lambda_{1} \int_{0}^{1} H(s, r) a_{1}(r)\right. \\
& \left.\times f_{1}(u(r), v(r)) d r\right) d s \\
& +\frac{t^{\alpha-1}}{\left(1-\Delta_{1}\right)} \sum_{i=1}^{m-2} a_{1 i} \\
& \times \int_{0}^{1} G_{1}\left(\xi_{1 i}, s\right) \phi_{q} \\
& \times\left(\lambda_{1} \int_{0}^{1} H(s, r) a_{1}(r)\right. \\
& \left.\left.\times f_{1}(u(r), v(r)) d r\right) d s\right\} \\
& \geq \int_{0}^{1} g(s) G(s, s) \phi_{q} \\
& \times\left(\lambda_{1} \int_{0}^{1} H(s, r) a_{1}(r) f_{1}(u(r), v(r)) d r\right) d s \\
& +\frac{\gamma^{\alpha-1}}{\left(1-\Delta_{1}\right)} \sum_{i=1}^{m-2} a_{1 i} \\
& \times \int_{0}^{1} G_{1}\left(\xi_{1 i}, s\right) \phi_{q} \\
& \times\left(\lambda_{1} \int_{0}^{1} H(s, r) a_{1}(r) f_{1}(u(r), v(r)) d r\right) d s \\
& \geq \eta \int_{\gamma}^{\delta} G(s, s) \phi_{q} \\
& \times\left(\lambda_{1} \int_{0}^{1} H(s, r) a_{1}(r) f_{1}(u(r), v(r)) d r\right) d s \\
& +\frac{\gamma^{\alpha-1}}{\left(1-\Delta_{1}\right)} \sum_{i=1}^{m-2} a_{1 i} \\
& \times \int_{\gamma}^{\delta} G_{1}\left(\xi_{1 i}, s\right) \phi_{q}
\end{aligned}
$$

$$
\begin{aligned}
& \quad \times\left(\lambda_{1} \int_{0}^{1} H(s, r) a_{1}(r) f_{1}(u(r), v(r)) d r\right) d s \\
& \geq \sigma\left[\int_{\gamma}^{\delta} G(s, s) \phi_{q}\right. \\
& \quad \times\left(\lambda_{1} \int_{0}^{1} H(s, r) a_{1}(r) f_{1}(u(r), v(r)) d r\right) d s \\
& +\frac{1}{\left(1-\Delta_{1}\right)} \sum_{i=1}^{m-2} a_{1 i} \\
& \quad \times \int_{\gamma}^{\delta} G_{1}\left(\xi_{1 i}, s\right) \phi_{q} \\
& \quad \times\left(\lambda_{1} \int_{0}^{1} H(s, r) a_{1}(r)\right. \\
& \geq \frac{\sigma}{3}\left\|T_{1}(u, v)\right\| .
\end{aligned}
$$

In the same way, for any $(u, v) \in K$, we have

$$
\min _{\gamma \leq t \leq \delta} T_{2}(u, v)(t) \geq \frac{\sigma}{3}\left\|T_{2}(u, v)\right\| .
$$

Therefore,

$$
\begin{gathered}
\min _{\gamma \leq t \leq \delta}\left(T_{1}(u, v)(t)+T_{2}(u, v)(t)\right) \\
\quad \geq \frac{\sigma}{3}\left\|T_{1}(u, v)\right\|+\frac{\sigma}{3}\left\|T_{2}(u, v)\right\| \\
\quad=\frac{\sigma}{3}\left\|\left(T_{1}(u, v), T_{2}(u, v)\right)\right\| .
\end{gathered}
$$

From the above, we conclude that $T(u, v)(t)=\left(T_{1}(u, v)(t)\right.$, $\left.T_{2}(u, v)(t)\right) \in K$, that is, $T(K) \subset K$. This completes the proof.

It is clear that the existence of a positive solution for system (6) is equivalent to the existence of a nontrivial fixed point of $T$ in $K$.

Theorem 15. Assume that (L1), (L2), and (L3) are satisfied. Then, system (6) and (7) has at least one positive solution.

Proof. It follows from Lemma 14 that $T: K \rightarrow K$. Furthermore, by the application of the Ascoli-Arzela theorem, which we omit, $T$ is a completely continuous operator.

Condition (L3) implies that there is $\epsilon>0$ sufficiently small such that

$$
\begin{aligned}
\phi_{q}\left(\lambda_{j}\right) \leq \frac{1}{2}\left[\int_{0}^{1} G(s, s) \phi_{q}\right. \\
\quad \times\left(\int_{0}^{1} H(s, r) a_{j}(r)\left(f_{j}^{*}+\epsilon\right) d r\right) d s \\
+\frac{1}{\left(1-\Delta_{j}\right)} \sum_{i=1}^{m-2} a_{j i}
\end{aligned}
$$




$$
\begin{aligned}
& \times \int_{0}^{1} G_{1}\left(\xi_{j i}, s\right) \phi_{q} \\
& \times\left(\int_{0}^{1} H(s, r) a_{j}(r)\right. \\
& \left.\left.\times\left(f_{j}^{*}+\epsilon\right) d r\right) d s\right]^{-1}, \\
& j=1,2 \text {, } \\
& \phi_{q}\left(\lambda_{j}\right) \geq \frac{3}{2 \sigma}\left[\int_{0}^{1} G(s, s) \phi_{q}\right. \\
& \times\left(\int_{\gamma}^{\delta} H(s, r) a_{j}(r)\left(f_{j}^{* *}-\epsilon\right) d r\right) d s \\
& +\frac{1}{\left(1-\Delta_{j}\right)} \sum_{i=1}^{m-2} a_{j i} \\
& \times \int_{0}^{1} G_{1}\left(\xi_{j i}, s\right) \phi_{q} \\
& \times\left(\int_{\gamma}^{\delta} H(s, r) a_{j}(r)\right. \\
& \left.\left.\times\left(f_{j}^{* *}-\epsilon\right) d r\right) d s\right]^{-1}, \\
& j=1,2 \text {. }
\end{aligned}
$$

Now given this $\epsilon$, it follows from condition (L1) that there exists some number $r_{1}^{*}>0$ such that

$$
f_{1}(u, v) \leq\left(f_{1}^{*}+\epsilon\right) \phi_{p}(u+v)
$$

whenever $\|(u, v)\|<r_{1}^{*}$. Similarly, by condition (L1), for the same $\epsilon$, there exists some number $r_{2}^{*}>0$ such that

$$
f_{2}(u, v) \leq\left(f_{2}^{*}+\epsilon\right) \phi_{p}(u+v)
$$

whenever $\|(u, v)\|<r_{2}^{*}$. In particular, by putting $r^{*}=$ $\min \left\{r_{1}^{*}, r_{2}^{*}\right\}$, we conclude that both (54) and (55) hold whenever $\|(u, v)\|<r^{*}$. So, define $\Omega_{1}$ by

$$
\Omega_{1}=\left\{(u, v) \in K:\|(u, v)\|<r^{*}\right\} .
$$

Then, for $(u, v) \in K \cap \partial \Omega_{1}$, we have

$$
\begin{aligned}
\left\|T_{1}(u, v)\right\|=\max _{0 \leq t \leq 1} T_{1}(u, v)(t) & \\
=\max _{0 \leq t \leq 1}\left\{\int_{0}^{1} G(t, s) \phi_{q}\right. & \\
& \times\left(\lambda_{1} \int_{0}^{1} H(s, r) a_{1}(r)\right. \\
& \left.\times f_{1}(u(r), v(r)) d r\right) d s
\end{aligned}
$$

$$
\begin{aligned}
& +\frac{t^{\alpha-1}}{\left(1-\Delta_{1}\right)} \sum_{i=1}^{m-2} a_{1 i} \\
& \times \int_{0}^{1} G_{1}\left(\xi_{1 i}, s\right) \phi_{q} \\
& \quad \times\left(\lambda_{1} \int_{0}^{1} H(s, r) a_{1}(r)\right.
\end{aligned}
$$$$
\left.\left.\times f_{1}(u(r), v(r)) d r\right) d s\right\}
$$$$
=\phi_{q}\left(\lambda_{1}\right)\left\{\int_{0}^{1} G(s, s) \phi_{q}\right.
$$$$
\times\left(\int_{0}^{1} H(s, r) a_{1}(r)\right.
$$$$
\left.\times f_{1}(u(r), v(r)) d r\right) d s
$$$$
+\frac{1}{\left(1-\Delta_{1}\right)} \sum_{i=1}^{m-2} a_{1 i}
$$$$
\times \int_{0}^{1} G_{1}\left(\xi_{1 i}, s\right) \phi_{q}
$$$$
\times\left(\int_{0}^{1} H(s, r) a_{1}(r)\right.
$$$$
\left.\left.\times f_{1}(u(r), v(r)) d r\right) d s\right\}
$$

$\leq \phi_{q}\left(\lambda_{1}\right)\left\{\int_{0}^{1} G(s, s) \phi_{q}\right.$

$$
\times\left(\int_{0}^{1} H(s, r) a_{1}(r)\left(f_{1}^{*}+\epsilon\right)\right.
$$$$
\left.\times \phi_{p}(u(r)+v(r)) d r\right) d s
$$$$
+\frac{1}{\left(1-\Delta_{1}\right)} \sum_{i=1}^{m-2} a_{1 i}
$$$$
\times \int_{0}^{1} G_{1}\left(\xi_{1 i}, s\right) \phi_{q}
$$$$
\times\left(\int_{0}^{1} H(s, r) a_{1}(r)\left(f_{1}^{*}+\epsilon\right)\right.
$$

$$
\left.\left.\times \phi_{p}(u(r)+v(r)) d r\right) d s\right\}
$$

$\leq \phi_{q}\left(\lambda_{1}\right)\left\{\int_{0}^{1} G(s, s) \phi_{q}\right.$

$$
\times\left(\int_{0}^{1} H(s, r) a_{1}(r)\left(f_{1}^{*}+\epsilon\right)\right.
$$

$\left.\times \phi_{p}(\|u\|+\|v\|) d r\right) d s$ 


$$
\begin{aligned}
& +\frac{1}{\left(1-\Delta_{1}\right)} \sum_{i=1}^{m-2} a_{1 i} \\
& \times \int_{0}^{1} G_{1}\left(\xi_{1 i}, s\right) \phi_{q} \\
& \times\left(\int_{0}^{1} H(s, r) a_{1}(r)\left(f_{1}^{*}+\epsilon\right) \phi_{p}\right. \\
& \times(\|u\|+\|v\|) d r) d s\} \\
& =\phi_{q}\left(\lambda_{1}\right)\left\{\int_{0}^{1} G(s, s) \phi_{q}\right. \\
& \times\left(\int_{0}^{1} H(s, r) a_{1}(r)\left(f_{1}^{*}+\epsilon\right) d r\right) \\
& \times(\|u\|+\|v\|) d s \\
& +\frac{1}{\left(1-\Delta_{1}\right)} \sum_{i=1}^{m-2} a_{1 i} \\
& \times \int_{0}^{1} G_{1}\left(\xi_{1 i}, s\right) \phi_{q} \\
& \times\left(\int_{0}^{1} H(s, r) a_{1}(r)\left(f_{1}^{*}+\epsilon\right) d r\right) \\
& \times(\|u\|+\|v\|) d s\} \\
& =\phi_{q}\left(\lambda_{1}\right)\left\{\int_{0}^{1} G(s, s) \phi_{q}\right. \\
& \times\left(\int_{0}^{1} H(s, r) a_{1}(r)\left(f_{1}^{*}+\epsilon\right) d r\right) d s \\
& +\frac{1}{\left(1-\Delta_{1}\right)} \sum_{i=1}^{m-2} a_{1 i} \\
& \times \int_{0}^{1} G_{1}\left(\xi_{1 i}, s\right) \phi_{q} \\
& \times\left(\int_{0}^{1} H(s, r) a_{1}(r)\right. \\
& \left.\left.\times\left(f_{1}^{*}+\epsilon\right) d r\right) d s\right\}
\end{aligned}
$$

$\cdot\|(u, v)\|$

$\leq \frac{1}{2}\|(u, v)\|$

Also, by similarly argument, we get

$$
\left\|T_{2}(u, v)\right\| \leq \frac{1}{2}\|(u, v)\|,
$$

for $(u, v) \in K \cap \partial \Omega_{1}$. Thus, for $(u, v) \in K \cap \partial \Omega_{1}$, we have

$$
\begin{aligned}
\|T(u, v)\| & =\left\|\left(T_{1}(u, v), T_{2}(u, v)\right)\right\| \\
& =\left\|T_{1}(u, v)\right\|+\left\|T_{2}(u, v)\right\| \\
& \leq \frac{1}{2}\|(u, v)\|+\frac{1}{2}\|(u, v)\|=\|(u, v)\| .
\end{aligned}
$$

On the other hand, letting $\epsilon>0$ be the same number selected at the beginning this proof, it follows from condition (L2) that there exists number $r^{* *}>0$ such that

$$
\begin{aligned}
& f_{1}(u, v) \geq\left(f_{1}^{* *}-\epsilon\right) \phi_{p}(u+v), \\
& f_{2}(u, v) \geq\left(f_{2}^{* *}-\epsilon\right) \phi_{p}(u+v),
\end{aligned}
$$

whenever $u+v \geq r^{* *}$. Let

$$
r=\max \left\{2 r^{*}, \frac{3 r^{* *}}{\sigma}\right\}
$$

Moreover, let

$$
\Omega_{2}=\{(u, v) \in K:\|(u, v)\|<r\} .
$$

Then, $\bar{\Omega}_{1} \subseteq \Omega_{2}$.

If $(u, v) \in K \cap \partial \Omega_{2}$, then it follows that for any $t \in[\gamma, \delta]$,

$$
u(t)+v(t) \geq \min _{t \in[\gamma, \delta]}[u(t)+v(t)] \geq \frac{\sigma}{3}\|(u, v)\| \geq r^{* *}
$$

Thus, (63) shows that for $(u, v) \in K \cap \partial \Omega_{2}$, (60) holds, whenever $t \in[\gamma, \delta]$.

So, for each $(u, v) \in K \cap \partial \Omega_{2}$, we have

$$
\begin{aligned}
\left\|T_{1}(u, v)\right\|=\max _{0 \leq t \leq 1} T_{1}(u, v)(t) & \\
=\phi_{q}\left(\lambda_{1}\right)\left\{\int_{0}^{1} G(s, s) \phi_{q}\right. & \\
& \times\left(\int_{0}^{1} H(s, r) a_{1}(r)\right. \\
& \left.\times f_{1}(u(r), v(r)) d r\right) d s \\
& +\frac{1}{\left(1-\Delta_{1}\right)} \sum_{i=1}^{m-2} a_{1 i} \\
& \times \int_{0}^{1} G_{1}\left(\xi_{1 i}, s\right) \phi_{q} \\
& \times\left(\int_{0}^{1} H(s, r) a_{1}(r)\right. \\
& \left.\left.\times f_{1}(u(r), v(r)) d r\right) d s\right\}
\end{aligned}
$$




$$
\begin{aligned}
& \geq \phi_{q}\left(\lambda_{1}\right)\left\{\int_{0}^{1} G(s, s) \phi_{q}\right. \\
& \times\left(\int_{\gamma}^{\delta} H(s, r) a_{1}(r)\right. \\
& \left.\times f_{1}(u(r), v(r)) d r\right) d s \\
& +\frac{1}{\left(1-\Delta_{1}\right)} \sum_{i=1}^{m-2} a_{1 i} \\
& \times \int_{0}^{1} G_{1}\left(\xi_{1 i}, s\right) \phi_{q} \\
& \times\left(\int_{\gamma}^{\delta} H(s, r) a_{1}(r)\right. \\
& \left.\left.\times f_{1}(u(r), v(r)) d r\right) d s\right\} \\
& \geq \phi_{q}\left(\lambda_{1}\right)\left\{\int_{0}^{1} G(s, s) \phi_{q}\right. \\
& \times\left(\int_{\gamma}^{\delta} H(s, r) a_{1}(r)\left(f_{1}^{* *}-\epsilon\right) \phi_{p}\right. \\
& \times(u(r)+v(r)) d r) d s \\
& +\frac{1}{\left(1-\Delta_{1}\right)} \sum_{i=1}^{m-2} a_{1 i} \\
& \times \int_{0}^{1} G_{1}\left(\xi_{1 i}, s\right) \phi_{q} \\
& \times\left(\int_{\gamma}^{\delta} H(s, r) a_{1}(r)\left(f_{1}^{* *}-\epsilon\right) \phi_{p}\right. \\
& \times(u(r)+v(r)) d r) d s\} \\
& \geq \phi_{q}\left(\lambda_{1}\right)\left\{\int_{0}^{1} G(s, s) \phi_{q}\right. \\
& \times\left(\int_{\gamma}^{\delta} H(s, r) a_{1}(r)\left(f_{1}^{* *}-\epsilon\right)\right. \\
& \left.\times \phi_{p}\left(\frac{\sigma}{3}\|(u, v)\|\right) d r\right) d s \\
& +\frac{1}{\left(1-\Delta_{1}\right)} \sum_{i=1}^{m-2} a_{1 i} \\
& \times \int_{0}^{1} G_{1}\left(\xi_{1 i}, s\right) \phi_{q} \\
& \times\left(\int_{\gamma}^{\delta} H(s, r) a_{1}(r)\left(f_{1}^{* *}-\epsilon\right)\right.
\end{aligned}
$$$$
\left.\left.\times \phi_{p}\left(\frac{\sigma}{3}\|(u, v)\|\right) d r\right) d s\right\}
$$$$
=\phi_{q}\left(\lambda_{1}\right) \cdot \frac{\sigma}{3}\left\{\int_{0}^{1} G(s, s) \phi_{q}\right.
$$$$
\times\left(\int_{\gamma}^{\delta} H(s, r) a_{1}(r)\right.
$$$$
\left.\times\left(f_{1}^{* *}-\epsilon\right) d r\right) d s
$$$$
+\frac{1}{\left(1-\Delta_{1}\right)} \sum_{i=1}^{m-2} a_{1 i}
$$$$
\times \int_{0}^{1} G_{1}\left(\xi_{1 i}, s\right) \phi_{q}
$$$$
\times\left(\int_{\gamma}^{\delta} H(s, r) a_{1}(r)\right.
$$$$
\left.\left.\times\left(f_{1}^{* *}-\epsilon\right) d r\right) d s\right\}
$$

$\cdot\|(u, v)\|$

$$
\geq \frac{1}{2}\|(u, v)\| \text {. }
$$

Similarly, we obtain that

$$
\left\|T_{2}(u, v)\right\| \geq \frac{1}{2}\|(u, v)\|
$$

for $(u, v) \in K \cap \partial \Omega_{2}$. Thus, for $(u, v) \in K \cap \partial \Omega_{2}$, we have

$$
\begin{aligned}
\|T(u, v)\| & =\left\|\left(T_{1}(u, v), T_{2}(u, v)\right)\right\| \\
& =\left\|T_{1}(u, v)\right\|+\left\|T_{2}(u, v)\right\| \\
& \geq \frac{1}{2}\|(u, v)\|+\frac{1}{2}\|(u, v)\|=\|(u, v)\| .
\end{aligned}
$$

Thus, all conditions of Theorem 13 are satisfied. Consequently, we conclude that $T$ has a fixed point on $K$. This is a positive solution of systems (6) and (7). The proof is completed.

\section{Existence of a Positive Solution: Case II}

In this section, we assume that $\gamma \in(0,1 / 4)$ and $\delta=1-\gamma$. We now provide a set of conditions under which the problem (6) and (8) will have at least one positive solution. we need conditions (L1) and (L2) in this section. furthermore, we use notations $\eta, \sigma$, and $K$ which were defined in Section 3. we will introduce new conditions. 
(L4) The functionals $\psi_{1}(u)$ and $\psi_{2}(v)$ are continuous in $u$ and $v$ and nonnegative for $u, v \geq 0$ and satisfy

$$
\begin{aligned}
& \lim _{\|u\| \rightarrow 0^{+}} \frac{\psi_{1}(u)}{\|u\|}=0, \\
& \lim _{\|v\| \rightarrow 0^{+}} \frac{\psi_{2}(v)}{\|v\|}=0 .
\end{aligned}
$$

(L5) There are numbers $N_{j}$ and $n_{j}$, where

$$
\begin{aligned}
& N_{j}=\frac{1}{4}\left[\int_{0}^{1} G(s, s) \phi_{q}\right. \\
& \times\left(\int_{0}^{1} H(s, r) a_{j}(r)\left(f_{j}^{*}\right) d r\right) d s \\
& +\frac{1}{\left(1-\Delta_{j}\right)} \sum_{i=1}^{m-2} a_{j i} \\
& \times \int_{0}^{1} G_{1}\left(\xi_{j i}, s\right) \phi_{q} \\
& \left.\times\left(\int_{0}^{1} H(s, r) a_{j}(r)\left(f_{j}^{*}\right) d r\right) d s\right]^{-1}, \\
& n_{j}=\frac{3}{2 \sigma}\left[\int_{0}^{1} G(s, s) \phi_{q}\right. \\
& \times\left(\int_{\gamma}^{\delta} H(s, r) a_{j}(r)\left(f_{j}^{* *}\right) d r\right) d s \\
& +\frac{1}{\left(1-\Delta_{j}\right)} \sum_{i=1}^{m-2} a_{j i} \\
& \times \int_{0}^{1} G_{1}\left(\xi_{j i}, s\right) \phi_{q} \\
& \left.\times\left(\int_{\gamma}^{\delta} H(s, r) a_{j}(r)\left(f_{j}^{* *}\right) d r\right) d s\right]^{-1},
\end{aligned}
$$

such that $\phi_{p}\left(n_{j}\right)<\lambda_{j}<\phi_{p}\left(N_{j}\right), j=1,2$.

Remark 16. Condition (67) in (L4) is true only if for each $\mu>$ 0 there is $r>0$ such that whenever $0<\|u\| \leq r$, it follows that $0<\psi_{1}(u) /\|u\|<\mu$. The same is true for condition (68) involving $\psi_{2}$.

By repeating the way that we used in Section 3, with a minor modification, we can get that $(u, v) \in C(0,1) \times C(0,1)$ is a solution of systems (6) and (8) if and only if $(u, v) \in$ $C[0,1] \times C[0,1]$ is a solution of the following nonlinear integral system:

$$
\begin{aligned}
u(t)= & \int_{0}^{1} G(t, s) \phi_{q} \\
& \times\left(\lambda_{1} \int_{0}^{1} H(s, r) a_{1}(r) f_{1}(u(r), v(r)) d r\right) d s \\
& +\frac{t^{\alpha-1}}{\left(1-\Delta_{1}\right)} \sum_{i=1}^{m-2} a_{1 i}
\end{aligned}
$$

$$
\begin{aligned}
& \times \int_{0}^{1} G_{1}\left(\xi_{1 i}, s\right) \phi_{q} \\
& \times\left(\lambda_{1} \int_{0}^{1} H(s, r) a_{1}(r)\right. \\
& \left.\times f_{1}(u(r), v(r)) d r\right) d s \\
& +\frac{\Gamma\left(\alpha-\beta_{1}\right) t^{\alpha-1}}{\left(1-\Delta_{1}\right) \Gamma(\alpha)} \psi_{1}(u), \\
& \int_{0}^{1} \frac{\Gamma(t, s) \phi_{q}\left(\lambda_{2} \int_{0}^{1} H(s, r)\right.}{\left(1-\Delta_{2}\right) \Gamma(\alpha)} \psi_{2}(v) . \\
& +\frac{t^{\alpha-1}}{\left(1-\Delta_{2}\right)} \sum_{i=1}^{m-2} a_{2 i} \\
& \left.\times \int_{0}^{1} G_{1}\left(\xi_{2 i}, s\right) \phi_{q}(r) f_{2}(u(r), v(r)) d r\right) d s \\
\times\left(\lambda_{2} \int_{0}^{1} H(s, r) a_{2}(r)\right. & \left.\times f_{2}(u(r), v(r)) d r\right) d s
\end{aligned}
$$

Thus, we define $S: E \rightarrow E$ defined by $S(u, v)=\left(S_{1}(u, v)\right.$, $\left.S_{2}(u, v)\right)$, where

$$
\begin{aligned}
S_{1}(u, v)= & \int_{0}^{1} G(t, s) \phi_{q} \\
& \times\left(\lambda_{1} \int_{0}^{1} H(s, r) a_{1}(r) f_{1}(u(r), v(r)) d r\right) d s \\
& +\frac{t^{\alpha-1}}{\left(1-\Delta_{1}\right)} \sum_{i=1}^{m-2} a_{1 i} \\
& \times \int_{0}^{1} G_{1}\left(\xi_{1 i}, s\right) \phi_{q} \\
& \times\left(\lambda_{1} \int_{0}^{1} H(s, r) a_{1}(r)\right. \\
& +\frac{\Gamma\left(\alpha-\beta_{1}\right) t^{\alpha-1}}{\left(1-\Delta_{1}\right) \Gamma(\alpha)} \psi_{1}(u),
\end{aligned}
$$




$$
\begin{aligned}
S_{2}(u, v)= & \int_{0}^{1} G(t, s) \phi_{q} \\
& \times\left(\lambda_{2} \int_{0}^{1} H(s, r) a_{2}(r) f_{2}(u(r), v(r)) d r\right) d s \\
& +\frac{t^{\alpha-1}}{\left(1-\Delta_{2}\right)} \sum_{i=1}^{m-2} a_{2 i} \\
& \times \int_{0}^{1} G_{1}\left(\xi_{2 i}, s\right) \phi_{q} \\
& \times\left(\lambda_{2} \int_{0}^{1} H(s, r) a_{2}(r)\right. \\
& +\frac{\Gamma\left(\alpha-\beta_{1}\right) t^{\alpha-1}}{\left(1-\Delta_{2}\right) \Gamma(\alpha)} \psi_{2}(v) .
\end{aligned}
$$

Lemma 17. Suppose that (H1), (H2), and (H3) hold. Then, the operator $S: K \rightarrow K$ is well defined, that is, $S(K) \subseteq K$.

Proof. For any $(u, v) \in K$, by (H1), (H2), (H3), and Lemma 11, $S_{1}(u, v)(t) \geq 0, S_{2}(u, v)(t) \geq 0, t \in[0,1]$, and it follows from (71) that

$$
\begin{aligned}
& \left\|S_{1}(u, v)\right\|=\int_{0}^{1} G(s, s) \phi_{q} \\
& \times\left(\lambda_{1} \int_{0}^{1} H(s, r) a_{1}(r)\right. \\
& \left.\times f_{1}(u(r), v(r)) d r\right) d s \\
& +\frac{1}{\left(1-\Delta_{1}\right)} \sum_{i=1}^{m-2} a_{1 i} \\
& \times \int_{0}^{1} G_{1}\left(\xi_{1 i}, s\right) \phi_{q} \\
& \times\left(\lambda_{1} \int_{0}^{1} H(s, r) a_{1}(r)\right. \\
& \left.\times f_{1}(u(r), v(r)) d r\right) d s \\
& +\frac{\Gamma\left(\alpha-\beta_{1}\right)}{\left(1-\Delta_{1}\right) \Gamma(\alpha)} \psi_{1}(u) \\
& =\left(\int_{0}^{\gamma}+\int_{\gamma}^{\delta}+\int_{\delta}^{1}\right) \\
& \times\left(G(s, s) \phi_{q}\right. \\
& \times\left(\lambda_{1} \int_{0}^{1} H(s, r) a_{1}(r)\right. \\
& \left.\left.\times f_{1}(u(r), v(r)) d r\right)\right) d s
\end{aligned}
$$

$$
\begin{aligned}
& +\frac{1}{\left(1-\Delta_{1}\right)} \Sigma_{i=1}^{m-2} a_{1 i} \\
& \times\left[\left(\int_{0}^{\gamma}+\int_{\gamma}^{\delta}+\int_{\delta}^{1}\right)\right. \\
& \times\left(G_{1}\left(\xi_{1 i}, s\right) \phi_{q}\right. \\
& \times\left(\lambda_{1} \int_{0}^{1} H(s, r) a_{1}(r)\right. \\
& +\frac{\Gamma\left(\alpha-\beta_{1}\right)}{\left(1-\Delta_{1}\right) \Gamma(\alpha)} \psi_{1}(u) \\
& \left.+\frac{\Gamma\left(\alpha-\beta_{1}\right)}{3\left(1-\Delta_{1}\right) \Gamma(\alpha)} \psi_{1}(u)\right] . \\
& \left.\left.\left.\left.\times \int_{\gamma}^{\delta} G_{1}(s, s) \phi_{q}(r)\right) d r\right)\right) d s\right] \\
& \times\left(\xi_{1 i}, s\right) \phi_{q}^{1} H(s, r) a_{1}(r) \\
& \times \frac{1}{\left(1-\Delta_{1}\right)} \sum_{i=1}^{m-2} a_{1 i} \\
& \left.\times f_{1}(u(r), v(r)) d r\right) d s
\end{aligned}
$$

Thus, for any $(u, v) \in K$, it follows from Lemma 11 and (73) that

$$
\begin{aligned}
& \min _{\gamma \leq t \leq \delta} S_{1}(u, v)(t) \\
&=\min _{\gamma \leq t \leq \delta}\left\{\int_{0}^{1} G(t, s) \phi_{q}\right. \\
& \quad \times\left(\lambda_{1} \int_{0}^{1} H(s, r) a_{1}(r)\right. \\
&\left.\quad \times f_{1}(u(r), v(r)) d r\right) d s \\
&+\frac{t^{\alpha-1}}{\left(1-\Delta_{1}\right)} \sum_{i=1}^{m-2} a_{1 i} \\
& \quad \times \int_{0}^{1} G_{1}\left(\xi_{1 i}, s\right) \phi_{q}
\end{aligned}
$$




$$
\begin{aligned}
& \times\left(\lambda_{1} \int_{0}^{1} H(s, r) a_{1}(r)\right. \\
& \left.\times f_{1}(u(r), v(r)) d r\right) d s \\
& \left.+\frac{\Gamma\left(\alpha-\beta_{1}\right) t^{\alpha-1}}{\left(1-\Delta_{1}\right) \Gamma(\alpha)} \psi_{1}(u)\right\} \\
& \geq \int_{0}^{1} g(s) G(s, s) \phi_{q} \\
& \times\left(\lambda_{1} \int_{0}^{1} H(s, r) a_{1}(r) f_{1}(u(r), v(r)) d r\right) d s \\
& +\frac{\gamma^{\alpha-1}}{\left(1-\Delta_{1}\right)} \Sigma_{i=1}^{m-2} a_{1 i} \\
& \times \int_{0}^{1} G_{1}\left(\xi_{1 i}, s\right) \phi_{q} \\
& \times\left(\lambda_{1} \int_{0}^{1} H(s, r) a_{1}(r) f_{1}(u(r), v(r)) d r\right) d s \\
& +\frac{\Gamma\left(\alpha-\beta_{1}\right) \gamma^{\alpha-1}}{\left(1-\Delta_{1}\right) \Gamma(\alpha)} \psi_{1}(u) \\
& \geq \eta \int_{\gamma}^{\delta} G(s, s) \phi_{q} \\
& \times\left(\lambda_{1} \int_{0}^{1} H(s, r) a_{1}(r) f_{1}(u(r), v(r)) d r\right) d s \\
& +\frac{\gamma^{\alpha-1}}{\left(1-\Delta_{1}\right)} \sum_{i=1}^{m-2} a_{1 i} \\
& \times \int_{\gamma}^{\delta} G_{1}\left(\xi_{1 i}, s\right) \phi_{q} \\
& \times\left(\lambda_{1} \int_{0}^{1} H(s, r) a_{1}(r) f_{1}(u(r), v(r)) d r\right) d s \\
& +\frac{\Gamma\left(\alpha-\beta_{1}\right) \gamma^{\alpha-1}}{3\left(1-\Delta_{1}\right) \Gamma(\alpha)} \psi_{1}(u) \\
& \geq \sigma\left[\int_{\gamma}^{\delta} G(s, s) \phi_{q}\right. \\
& \times\left(\lambda_{1} \int_{0}^{1} H(s, r) a_{1}(r) f_{1}(u(r), v(r)) d r\right) d s \\
& +\frac{1}{\left(1-\Delta_{1}\right)} \Sigma_{i=1}^{m-2} a_{1 i} \\
& \times \int_{\gamma}^{\delta} G_{1}\left(\xi_{1 i}, s\right) \phi_{q} \\
& \times\left(\lambda_{1} \int_{0}^{1} H(s, r) a_{1}(r) f_{1}(u(r), v(r)) d r\right) d s
\end{aligned}
$$$$
\begin{gathered}
\left.+\frac{\Gamma\left(\alpha-\beta_{1}\right)}{3\left(1-\Delta_{1}\right) \Gamma(\alpha)} \psi_{1}(u)\right] \\
\geq \frac{\sigma}{3}\left\|S_{1}(u, v)\right\| .
\end{gathered}
$$

In the same way, for any $(u, v) \in K$, we have

$$
\min _{\gamma \leq t \leq \delta} S_{2}(u, v)(t) \geq \frac{\sigma}{3}\left\|S_{2}(u, v)\right\| .
$$

Therefore,

$$
\begin{aligned}
\min _{\gamma \leq t \leq \delta} & \left(S_{1}(u, v)(t)+S_{2}(u, v)(t)\right) \\
& \geq \frac{\sigma}{3}\left\|S_{1}(u, v)\right\|+\frac{\sigma}{3}\left\|S_{2}(u, v)\right\| \\
& =\frac{\sigma}{3}\left\|\left(S_{1}(u, v), S_{2}(u, v)\right)\right\| .
\end{aligned}
$$

From the above, we conclude that $S(u, v)(t)=\left(S_{1}(u, v)(t)\right.$, $\left.S_{2}(u, v)(t)\right) \in K$, that is, $S(K) \subset K$. This completes the proof.

It is obvious that existence of a solution for the problem (6) and (8) is equivalent to existence of a fixed point for $S$ on K.

Theorem 18. Suppose that conditions (L1), (L2), (L4), and (L5) hold. Then, systems (6) and (8) has at least one positive solution.

Proof. Lemma 17 shows that $S: K \rightarrow K$. Moreover, from continuity of $\psi_{1}, \psi_{2}, G$, and $f$, it is obvious that both $S_{1}$ and $S_{2}$ are completely continuous operators by the application of the Ascoli-Arzela theorem. Then, $S$ is a completely continuous operator. Also, condition (L3) implies that there is $\epsilon>0$ sufficiently small such that

$$
\begin{aligned}
\phi_{q}\left(\lambda_{j}\right) \leq \frac{1}{4}\left[\int_{0}^{1} G(s, s) \phi_{q}\right. \\
\quad \times\left(\int_{0}^{1} H(s, r) a_{j}(r)\left(f_{j}^{*}+\epsilon\right) d r\right) d s \\
+\frac{1}{\left(1-\Delta_{j}\right)} \sum_{i=1}^{m-2} a_{j i} \\
\quad \times \int_{0}^{1} G_{1}\left(\xi_{j i}, s\right) \phi_{q} \\
\quad \times\left(\int_{0}^{1} H(s, r) a_{j}(r)\right. \\
\left.\left.\quad \times\left(f_{j}^{*}+\epsilon\right) d r\right) d s\right]^{-1}, \\
\quad j=1,2,
\end{aligned}
$$




$$
\begin{aligned}
& \phi_{q}\left(\lambda_{j}\right) \geq \frac{3}{2 \sigma}\left[\int_{0}^{1} G(s, s) \phi_{q}\right. \\
& \times\left(\int_{\gamma}^{\delta} H(s, r) a_{j}(r)\left(f_{j}^{* *}-\epsilon\right) d r\right) d s \\
&+\frac{1}{\left(1-\Delta_{j}\right)} \sum_{i=1}^{m-2} a_{j i} \\
& \times \int_{0}^{1} G_{1}\left(\xi_{j i}, s\right) \phi_{q} \\
& \times\left(\int_{\gamma}^{\delta} H(s, r) a_{j}(r)\right. \\
&\left.\left.\times\left(f_{j}^{* *}-\epsilon\right) d r\right) d s\right]^{-1}
\end{aligned}
$$

Now given this $\epsilon$, just as before, conditions (54) and (55) remain true whenever $\|(u, v)\|<r^{*}$, exactly as in the proof of Theorem 15. It follows from (L4) and Remark 16 that there is $r_{1}^{* * *}$ such that $\psi_{1}(u) \leq \mu\|u\|$ whenever $0<\|u\| \leq r_{1}^{* * *}$, and there is $r_{2}^{* * *}$ such that $\psi_{2}(v) \leq \mu\|v\|$ whenever $0<\|v\| \leq r_{2}^{* * *}$. In particular and without loss of generality, let us suppose that

$$
0<\mu<\frac{\left(1-\Delta_{j}\right) \Gamma(\alpha)}{2 \Gamma\left(\alpha-\beta_{1}\right)}, \quad j=1,2 .
$$

Now, let $r_{1}=\min \left\{r^{*}, r_{1}^{* * *}, r_{2}^{* * *}\right\}$. Observe that for any $(u, v) \in K$ we have that $\|u\|,\|v\| \leq\|(u, v)\|$. Then, we obtain that for all $(u, v) \in K$ satisfying $0<\|(u, v)\|<r_{1}$ we have

$$
\begin{aligned}
& f_{1}(u, v) \leq\left(f_{1}^{*}+\epsilon\right) \phi_{p}(u+v), \\
& f_{2}(u, v) \leq\left(f_{2}^{*}+\epsilon\right) \phi_{p}(u+v), \\
& \psi_{1}(u) \leq \frac{\left(1-\Delta_{1}\right) \Gamma(\alpha)}{2 \Gamma\left(\alpha-\beta_{1}\right)}\|u\|, \\
& \psi_{2}(v) \leq \frac{\left(1-\Delta_{2}\right) \Gamma(\alpha)}{2 \Gamma\left(\alpha-\beta_{1}\right)}\|v\| .
\end{aligned}
$$

So, define $\Omega_{1}$ by $\Omega_{1}=\left\{(u, v) \in K: 0<\|(u, v)\|<r_{1}\right\}$. We obtain for $(u, v) \in K \cap \partial \Omega_{1}$ that

$$
\begin{aligned}
\left\|S_{1}(u, v)\right\|=\max _{0 \leq t \leq 1} S_{1}(u, v)(t) & \\
=\max _{0 \leq t \leq 1}\left\{\int_{0}^{1} G(t, s) \phi_{q}\right. & \\
& \times\left(\lambda_{1} \int_{0}^{1} H(s, r) a_{1}(r)\right. \\
& \left.\times f_{1}(u(r), v(r)) d r\right) d s
\end{aligned}
$$

$$
\begin{aligned}
& +\frac{t^{\alpha-1}}{\left(1-\Delta_{1}\right)} \sum_{i=1}^{m-2} a_{1 i} \\
& \times \int_{0}^{1} G_{1}\left(\xi_{1 i}, s\right) \phi_{q} \\
& \times\left(\lambda_{1} \int_{0}^{1} H(s, r) a_{1}(r)\right. \\
& \left.\times f_{1}(u(r), v(r)) d r\right) d s \\
& \left.+\frac{t^{\alpha-1} \Gamma\left(\alpha-\beta_{1}\right)}{\Gamma(\alpha)\left(1-\Delta_{1}\right)} \psi_{1}(u)\right\} \\
& \leq \phi_{q}\left(\lambda_{1}\right)\left\{\int_{0}^{1} G(s, s) \phi_{q}\right. \\
& \times\left(\int_{0}^{1} H(s, r) a_{1}(r)\right. \\
& \left.\times f_{1}(u(r), v(r)) d r\right) d s \\
& +\frac{1}{\left(1-\Delta_{1}\right)} \sum_{i=1}^{m-2} a_{1 i} \\
& \times \int_{0}^{1} G_{1}\left(\xi_{1 i}, s\right) \\
& \times \phi_{q}\left(\int_{0}^{1} H(s, r) a_{1}(r)\right. \\
& \left.\left.\times f_{1}(u(r), v(r)) d r\right) d s\right\} \\
& +\frac{\Gamma\left(\alpha-\beta_{1}\right)}{\Gamma(\alpha)\left(1-\Delta_{1}\right)} \psi_{1}(u) \\
& \leq \phi_{q}\left(\lambda_{1}\right)\left\{\int_{0}^{1} G(s, s) \phi_{q}\right. \\
& \times\left(\int_{0}^{1} H(s, r) a_{1}(r)\left(f_{1}^{*}+\epsilon\right)\right. \\
& \left.\times \phi_{p}(u(r)+v(r)) d r\right) d s \\
& +\frac{1}{\left(1-\Delta_{1}\right)} \sum_{i=1}^{m-2} a_{1 i} \\
& \times \int_{0}^{1} G_{1}\left(\xi_{1 i}, s\right) \phi_{q} \\
& \times\left(\int_{0}^{1} H(s, r) a_{1}(r)\left(f_{1}^{*}+\epsilon\right)\right. \\
& \left.\left.\times \phi_{p}(u(r)+v(r)) d r\right) d s\right\} \\
& +\frac{1}{2}\|u\|
\end{aligned}
$$




$$
\begin{aligned}
& \leq \phi_{q}\left(\lambda_{1}\right)\left\{\int_{0}^{1} G(s, s) \phi_{q}\right. \\
& \times\left(\int_{0}^{1} H(s, r) a_{1}(r)\left(f_{1}^{*}+\epsilon\right)\right. \\
& \left.\times \phi_{p}(\|u\|+\|v\|) d r\right) d s \\
& +\frac{1}{\left(1-\Delta_{1}\right)} \sum_{i=1}^{m-2} a_{1 i} \\
& \times \int_{0}^{1} G_{1}\left(\xi_{1 i}, s\right) \phi_{q} \\
& \times\left(\int_{0}^{1} H(s, r) a_{1}(r)\left(f_{1}^{*}+\epsilon\right) \phi_{p}\right. \\
& \times(\|u\|+\|v\|) d r) d s\} \\
& +\frac{1}{2}\|u\| \\
& =\phi_{q}\left(\lambda_{1}\right)\left\{\int_{0}^{1} G(s, s) \phi_{q}\right. \\
& \times\left(\int_{0}^{1} H(s, r) a_{1}(r)\right. \\
& \left.\times\left(f_{1}^{*}+\epsilon\right) d r\right)(\|u\|+\|v\|) d s \\
& +\frac{1}{\left(1-\Delta_{1}\right)} \sum_{i=1}^{m-2} a_{1 i} \\
& \times \int_{0}^{1} G_{1}\left(\xi_{1 i}, s\right) \phi_{q} \\
& \times\left(\int_{0}^{1} H(s, r) a_{1}(r)\left(f_{1}^{*}+\epsilon\right) d r\right) \\
& \times(\|u\|+\|v\|) d s\} \\
& +\frac{1}{2}\|u\| \\
& =\phi_{q}\left(\lambda_{1}\right)\left\{\int_{0}^{1} G(s, s) \phi_{q}\right. \\
& \times\left(\int_{0}^{1} H(s, r) a_{1}(r)\left(f_{1}^{*}+\epsilon\right) d r\right) d s \\
& +\frac{1}{\left(1-\Delta_{1}\right)} \sum_{i=1}^{m-2} a_{1 i} \\
& \times \int_{0}^{1} G_{1}\left(\xi_{1 i}, s\right) \phi_{q} \\
& \times\left(\int_{0}^{1} H(s, r) a_{1}(r)\right. \\
& \left.\left.\times\left(f_{1}^{*}+\epsilon\right) d r\right) d s\right\}
\end{aligned}
$$$$
\cdot\|(u, v)\|+\frac{1}{2}\|u\|
$$$$
\leq \frac{1}{4}\|(u, v)\|+\frac{1}{2}\|u\| \text {. }
$$

Also, by similarly argument, we obtain that

$$
\left\|S_{2}(u, v)\right\| \leq \frac{1}{4}\|(u, v)\|+\frac{1}{2}\|v\|
$$

for $(u, v) \in K \cap \partial \Omega_{1}$. Thus, for $(u, v) \in K \cap \partial \Omega_{1}$, we have

$$
\begin{aligned}
\|S(u, v)\| & =\left\|\left(S_{1}(u, v), S_{2}(u, v)\right)\right\| \\
& =\left\|S_{1}(u, v)\right\|+\left\|S_{2}(u, v)\right\| \\
& \leq \frac{1}{4}\|(u, v)\|+\frac{1}{4}\|(u, v)\|+\frac{1}{2}\|u\|+\frac{1}{2}\|v\| \\
& =\frac{1}{2}\|(u, v)\|+\frac{1}{2}(\|u\|+\|v\|) \\
& =\frac{1}{2}\|(u, v)\|+\frac{1}{2}\|(u, v)\|=\|(u, v)\| .
\end{aligned}
$$

This implies that for $(u, v) \in K \cap \partial \Omega_{1}$, we have

$$
\|S(u, v)\| \leq\|(u, v)\| .
$$

On the other hand, letting $\epsilon>0$ be the same number selected at the beginning this proof, as before, condition (L2) implies that there exists number $r^{* *}>0$ such that

$$
\begin{aligned}
& f_{1}(u, v) \geq\left(f_{1}^{* *}-\epsilon\right) \phi_{p}(u+v), \\
& f_{2}(u, v) \geq\left(f_{2}^{* *}-\epsilon\right) \phi_{p}(u+v),
\end{aligned}
$$

whenever $u+v \geq r^{* *}$. Let

$$
r_{2}=\max \left\{2 r_{1}, \frac{3 r^{* *}}{\sigma}\right\} .
$$

Moreover, if we let

$$
\Omega_{2}=\left\{(u, v) \in K:\|(u, v)\|<r_{2}\right\},
$$

then, $\bar{\Omega}_{1} \subseteq \Omega_{2}$. 
Abstract and Applied Analysis

17

If $(u, v) \in K \cap \partial \Omega_{2}$, then, it follows that for any $t \in[\gamma, \delta]$,

$$
\begin{aligned}
u(t)+v(t) & \geq \min _{t \in[\gamma, \delta]}[u(t)+v(t)] \\
& \geq \frac{\sigma}{3}\|(u, v)\| \geq r^{* *} .
\end{aligned}
$$

Thus, (87) shows that for $(u, v) \in K \cap \partial \Omega_{2}$, (84) holds, whenever $t \in[\gamma, \delta]$.

In addition, recall that by condition (L4), $\psi_{1}$ and $\psi_{2}$ are assumed to be nonnegative for $(u, v) \in K$. So, for each $(u, v) \in$ $K \cap \partial \Omega_{2}$, we have

$$
\begin{aligned}
&\left\|S_{1}(u, v)\right\|=\max _{0 \leq t \leq 1} S_{1}(u, v)(t) \\
&=\phi_{q}\left(\lambda_{1}\right)\left\{\int_{0}^{1} G(s, s) \phi_{q}\right. \\
& \quad \times\left(\int_{0}^{1} H(s, r) a_{1}(r)\right. \\
&\left.\quad \times f_{1}(u(r), v(r)) d r\right) d s \\
&+\frac{1}{\left(1-\Delta_{1}\right)} \sum_{i=1}^{m-2} a_{1 i} \\
& \quad \times \int_{0}^{1} G_{1}\left(\xi_{1 i}, s\right) \phi_{q} \\
& \quad \times\left(\int_{0}^{1} H(s, r) a_{1}(r)\right. \\
&\left.\left.\quad \times f_{1}(u(r), v(r)) d r\right) d s\right\}
\end{aligned}
$$$$
+\frac{\Gamma\left(\alpha-\beta_{1}\right)}{\Gamma(\alpha)\left(1-\Delta_{1}\right)} \psi_{1}(u)
$$$$
\geq \phi_{q}\left(\lambda_{1}\right)\left\{\int_{0}^{1} G(s, s) \phi_{q}\right.
$$$$
\times\left(\int_{\gamma}^{\delta} H(s, r) a_{1}(r)\right.
$$$$
\left.\times f_{1}(u(r), v(r)) d r\right) d s
$$

$$
\begin{aligned}
& +\frac{1}{\left(1-\Delta_{1}\right)} \sum_{i=1}^{m-2} a_{1 i} \\
& \times \int_{0}^{1} G_{1}\left(\xi_{1 i}, s\right) \phi_{q} \\
& \quad \times\left(\int_{\gamma}^{\delta} H(s, r) a_{1}(r)\right. \\
& \left.\left.\quad \times f_{1}(u(r), v(r)) d r\right) d s\right\}
\end{aligned}
$$

$$
\begin{aligned}
& \geq \phi_{q}\left(\lambda_{1}\right)\left\{\int_{0}^{1} G(s, s) \phi_{q}\right. \\
& \quad \times\left(\int_{\gamma}^{\delta} H(s, r) a_{1}(r)\left(f_{1}^{* *}-\epsilon\right)\right. \\
&\left.\quad \times \phi_{p}(u(r)+v(r)) d r\right) d s
\end{aligned}
$$

$$
\begin{aligned}
& +\frac{1}{\left(1-\Delta_{1}\right)} \sum_{i=1}^{m-2} a_{1 i} \\
& \times \int_{0}^{1} G_{1}\left(\xi_{1 i}, s\right) \phi_{q} \\
& \quad \times\left(\int_{\gamma}^{\delta} H(s, r) a_{1}(r)\left(f_{1}^{* *}-\epsilon\right) \phi_{p}\right.
\end{aligned}
$$$$
\times(u(r)+v(r)) d r) d s\}
$$

$\geq \phi_{q}\left(\lambda_{1}\right)\left\{\int_{0}^{1} G(s, s) \phi_{q}\right.$

$$
\times\left(\int_{\gamma}^{\delta} H(s, r) a_{1}(r)\left(f_{1}^{* *}-\epsilon\right) \phi_{p}\right.
$$$$
\left.\times\left(\frac{\sigma}{3}\|u, v\|\right) d r\right) d s
$$

$$
\begin{aligned}
& +\frac{1}{\left(1-\Delta_{1}\right)} \sum_{i=1}^{m-2} a_{1 i} \\
& \times \int_{0}^{1} G_{1}\left(\xi_{1 i}, s\right) \phi_{q} \\
& \quad \times\left(\int_{\gamma}^{\delta} H(s, r) a_{1}(r)\left(f_{1}^{* *}-\epsilon\right)\right.
\end{aligned}
$$

$$
\left.\left.\times \phi_{p}\left(\frac{\sigma}{3}\|u, v\|\right) d r\right) d s\right\}
$$

$=\phi_{q}\left(\lambda_{1}\right) \cdot \frac{\sigma}{3}\left\{\int_{0}^{1} G(s, s) \phi_{q}\right.$

$$
\times\left(\int_{\gamma}^{\delta} H(s, r) a_{1}(r)\right.
$$$$
\left.\times\left(f_{1}^{* *}-\epsilon\right) d r\right) d s
$$

$+\frac{1}{\left(1-\Delta_{1}\right)} \sum_{i=1}^{m-2} a_{1 i}$

$\times \int_{0}^{1} G_{1}\left(\xi_{1 i}, s\right) \phi_{q}$ 


$$
\begin{aligned}
\times\left(\int_{\gamma}^{\delta} H(s, r) a_{1}(r)\right. \\
\left.\left.\quad \times\left(f_{1}^{* *}-\epsilon\right) d r\right) d s\right\}
\end{aligned}
$$

$$
\begin{gathered}
\cdot\|(u, v)\| \\
\geq \frac{1}{2}\|(u, v)\| .
\end{gathered}
$$

Similarly, one can get

$$
\left\|S_{2}(u, v)\right\| \geq \frac{1}{2}\|(u, v)\|,
$$

for $(u, v) \in K \cap \partial \Omega_{2}$. Thus, for $(u, v) \in K \cap \partial \Omega_{2}$, we have

$$
\begin{aligned}
\|S(u, v)\| & =\left\|\left(S_{1}(u, v), S_{2}(u, v)\right)\right\| \\
& =\left\|S_{1}(u, v)\right\|+\left\|S_{2}(u, v)\right\| \\
& \geq \frac{1}{2}\|(u, v)\|+\frac{1}{2}\|(u, v)\|=\|(u, v)\| .
\end{aligned}
$$

Thus, all conditions of Theorem 13 are satisfied. Consequently, we conclude that $S$ has a fixed point on $K$. This is a positive solution of systems (6) and (8). The proof is completed.

\section{Application}

Example 19. Consider the following singular boundary value problem:

$$
\begin{array}{r}
D_{0^{+}}^{3 / 2} \phi_{p}\left(D_{0^{+}}^{3 / 2} u\right)(t)=\lambda_{1} a_{1}(t) f_{1}(u(t), v(t)), \\
t \in(0,1), \\
D_{0^{+}}^{3 / 2} \phi_{p}\left(D_{0^{+}}^{3 / 2} v\right)(t)=\lambda_{2} a_{2}(t) f_{2}(u(t), v(t)), \\
t \in(0,1),
\end{array}
$$

subject to the boundary conditions

$$
\begin{aligned}
& D_{0^{+}}^{3 / 2} u(0)=D_{0^{+}}^{3 / 2} u(1)=0, \quad u(0)=0, \\
& D_{0^{+}}^{1 / 2} u(1)-\frac{1}{2} D_{0^{+}}^{1 / 2} u\left(\frac{1}{4}\right)-\frac{1}{3} D_{0^{+}}^{1 / 2} u\left(\frac{1}{2}\right)=0, \\
& D_{0^{+}}^{3 / 2} v(0)=D_{0^{+}}^{3 / 2} v(1)=0, \quad v(0)=0, \\
& D_{0^{+}}^{1 / 2} v(1)-\frac{3}{4} D_{0^{+}}^{1 / 2} v\left(\frac{1}{3}\right)-\frac{1}{5} D_{0^{+}}^{1 / 2} v\left(\frac{2}{3}\right)=0 .
\end{aligned}
$$

Here, $\alpha=\beta=3 / 2, \beta_{1}=1 / 2, p=2, m=4, a_{11}=1 / 2$, $a_{12}=1 / 3, a_{21}=3 / 4, a_{22}=1 / 5, \xi_{11}=1 / 4, \xi_{12}=1 / 2, \xi_{21}=1 / 3$ and $\xi_{22}=2 / 3$. Now, we have $q=2$,

$$
\begin{gathered}
f_{1}(u, v)=(u+v)\left(500-\frac{495}{u^{2}+v^{2}+1}\right), \\
f_{2}(u, v)=(u+v)\left(1000-\frac{990}{u^{2}+v^{2}+1}\right), \\
a_{1}(t)=t, \quad a_{2}(t)=2 t .
\end{gathered}
$$

It is easy to check that $f_{1}, f_{2}:[0, \infty) \times[0, \infty) \rightarrow$ $[0, \infty)$ are continuous. The functions $a_{1}(t)$ and $a_{2}(t)$ are obviously nonnegative for all $t \in[0,1]$. We now check that all conditions of Theorem 15 hold. By definition of functions $f_{1}$ and $f_{2}$, we get

$$
\begin{aligned}
& \lim _{(u, v) \rightarrow\left(0^{+}, 0^{+}\right)} \frac{f_{1}(u, v)}{\phi_{p}(u+v)} \\
& =\lim _{(u, v) \rightarrow\left(0^{+}, 0^{+}\right)}\left(500-\frac{495}{u^{2}+v^{2}+1}\right)=5, \\
& \lim _{(u, v) \rightarrow\left(0^{+}, 0^{+}\right)} \frac{f_{2}(u, v)}{\phi_{p}(u+v)} \\
& =\lim _{(u, v) \rightarrow\left(0^{+}, 0^{+}\right)}\left(1000-\frac{990}{u^{2}+v^{2}+1}\right)=10 .
\end{aligned}
$$

Thus, let

$$
f_{1}^{*}=5, \quad f_{2}^{*}=10 .
$$

On the other hand, we have

$$
\begin{aligned}
& \lim _{(u, v) \rightarrow(\infty, \infty)} \frac{f_{1}(u, v)}{\phi_{p}(u+v)} \\
& =\lim _{(u, v) \rightarrow(\infty, \infty)}\left(500-\frac{495}{u^{2}+v^{2}+1}\right)=500, \\
& \lim _{(u, v) \rightarrow(\infty, \infty)} \frac{f_{2}(u, v)}{\phi_{p}(u+v)} \\
& =\lim _{(u, v) \rightarrow(\infty, \infty)}\left(1000-\frac{990}{u^{2}+v^{2}+1}\right)=1000 .
\end{aligned}
$$

Thus, let

$$
f_{1}^{* *}=500, \quad f_{2}^{* *}=1000 .
$$

It follows from (94)-(97) that conditions (L1) and (L2) hold.

Choose $\gamma=1 / 4, \delta=3 / 4$. Then, by direct calculations, we can obtain that $\eta=0.3780, \sigma=0.3536$, and

$$
\begin{array}{ll}
\Delta_{1}=0.8333, & \Delta_{2}=0.9500, \\
m_{1}=0.0168, & m_{2}=0.0014, \\
M_{1}=0.1976, & M_{2}=0.0164 .
\end{array}
$$

Then, for $0.0168<\lambda_{1}<0.1976$ and $0.0014<$ $\lambda_{2}<0.0164$, condition (L3) holds. Thus, all conditions of Theorem 15 hold. Hence, system (91) with boundary conditions (92) has at least one positive solution.

Now, consider the problem (91) with following boundary conditions:

$D_{0^{+}}^{3 / 2} u(0)=D_{0^{+}}^{3 / 2} u(1)=0, \quad u(0)=0$,

$D_{0^{+}}^{1 / 2} u(1)-\frac{1}{2} D_{0^{+}}^{1 / 2} u\left(\frac{1}{4}\right)-\frac{1}{3} D_{0^{+}}^{1 / 2} u\left(\frac{1}{2}\right)=\psi_{1}(u)$,

$D_{0^{+}}^{3 / 2} v(0)=D_{0^{+}}^{3 / 2} v(1)=0, \quad v(0)=0$,

$D_{0^{+}}^{1 / 2} v(1)-\frac{3}{4} D_{0^{+}}^{1 / 2} v\left(\frac{1}{3}\right)-\frac{1}{5} D_{0^{+}}^{1 / 2} v\left(\frac{2}{3}\right)=\psi_{2}(v)$, 
where

$$
\begin{aligned}
& \psi_{1}(u)=\left(u\left(\frac{1}{4}\right)\right)^{2}, \\
& \psi_{2}(v)=\left(v\left(\frac{3}{4}\right)\right)^{3} .
\end{aligned}
$$

In this case, we check that all conditions of Theorem 18 hold. It follows from (94)-(97) that conditions (L1) and (L2) hold. We now show that (L4) and (L5) hold:

$$
\begin{aligned}
& \lim _{\|u\| \rightarrow 0^{+}} \frac{\psi_{1}(u)}{\|u\|}=\lim _{\|u\| \rightarrow 0^{+}} \frac{(u(1 / 4))^{2}}{\|u\|} \\
& \leq \lim _{\|u\| \rightarrow 0^{+}} \frac{\|u\|^{2}}{\|u\|}=\lim _{\|u\| \rightarrow 0^{+}}\|u\|=0, \\
& \lim _{\|v\| \rightarrow 0^{+}} \frac{\psi_{2}(v)}{\|v\|}=\lim _{\|v\| \rightarrow 0^{+}} \frac{(v(3 / 4))^{3}}{\|v\|} \\
& \leq \lim _{\|v\| \rightarrow 0^{+}} \frac{\|v\|^{3}}{\|v\|}=\lim _{\|v\| \rightarrow 0^{+}}\|v\|^{2}=0 .
\end{aligned}
$$

So, condition (L4) is satisfied. Now, by direct calculation, one can get

$$
\begin{array}{ll}
n_{1}=0.0168, & n_{2}=0.0014, \\
N_{1}=0.0998, & N_{2}=0.0082 .
\end{array}
$$

Then, for $0.0168<\lambda_{1}<0.0998$ and $0.0014<\lambda_{2}<0.0082$, condition (L5) holds. Thus, all conditions of Theorem 18 hold. Hence, system (91) with the boundary conditions (99) has at least one positive solution.

\section{Acknowledgment}

The authors would like to thank the anonymous referees for their valuable suggestions and comments.

\section{References}

[1] R. Hilfer, Applications of Fractional Calculus in Physics, World Scientific Publishing Co., River Edge, NJ, USA, 2000.

[2] J. Sabatier, O. P. Agrawal, and J. A. Tenreiro Machado, Advances in Fractional Calculus, Springer, Dordrecht, The Netherlands, 2007.

[3] A. A. Kilbas and J. J. Trujillo, "Differential equations of fractional order: methods, results and problems. I," Applicable Analysis, vol. 78, no. 1-2, pp. 153-192, 2001.

[4] A. A. Kilbas and J. J. Trujillo, "Differential equations of fractional order: methods, results and problems. II," Applicable Analysis, vol. 81, no. 2, pp. 435-493, 2002.

[5] A. Babakhani and V. Daftardar-Gejji, "Existence of positive solutions of nonlinear fractional differential equations," Journal of Mathematical Analysis and Applications, vol. 278, no. 2, pp. 434-442, 2003.

[6] D. Delbosco and L. Rodino, "Existence and uniqueness for a nonlinear fractional differential equation," Journal of Mathematical Analysis and Applications, vol. 204, no. 2, pp. 609-625, 1996.
[7] D. Baleanu, K. Diethelm, E. Scalas, and J. J. Trujillo, Fractional Calculus: Models and Numerical Methods, vol. 3 of Series on Complexity, Nonlinearity and Chaos, World Scientific Publishing, Hackensack, NJ, USA, 2012.

[8] D. Băleanu, O. G. Mustafa, and R. P. Agarwal, "On the solution set for a class of sequential fractional differential equations," Journal of Physics A, vol. 43, no. 38, Article ID 385209, 7 pages, 2010.

[9] D. Baleanu, H. Mohammadi, and Sh. Rezapour, "Some existence results on nonlinear fractional differential equations," Philosophical Transactions of the Royal Society A, vol. 371, no. 1990, 2013.

[10] H. Scher and E. W. Montroll, "Anomalous transit-time dispersion in amorphous solids," Physical Review B, vol. 12, no. 6, pp. 2455-2477, 1975.

[11] K. Diethelm and A. D. Freed, "On the solution of nonlinear fractional order differential equations used in the modeling of viscoplasticity," in Scientific Computing in Chemical Engineering II: Computational Fluid Dynamics, Reaction Engineering, and Molecular Properties, F. Keil, W. Mackens, H. voss, and J. Werther, Eds., Springer, Heidelberg, Germany, 1999.

[12] L. Gaul, P. Klein, and S. Kemple, "Damping description involving fractional operators," Mechanical Systems and Signal Processing, vol. 5, pp. 81-88, 1991.

[13] W. G. Glockle and T. F. Nonnenmacher, "A fractional calculus approach to self-semilar protein dynamics," Biophysical Journal, vol. 68, no. 1, pp. 46-53, 1995.

[14] F. Mainardi, "Fractional calculus: some basic problems in continuum and statistical mechanics," in Fractals and Fractional Calculus in Continuum Mechanics (Udine, 1996), A. Carpinteri and F. Mainardi, Eds., vol. 378 of CISM Courses and Lectures, pp. 291-348, Springer, New York, NY, USA, 1997.

[15] R. Metzler, W. Schick, H. G. Kilian, and T. F. Nonnenmacher, "Relaxation in filled polymers: a fractional calculus approach," Journal of Chemical Physics, vol. 103, pp. 7180-7186, 1995.

[16] Z. Bai and H. Lü, "Positive solutions for boundary value problem of nonlinear fractional differential equation," Journal of Mathematical Analysis and Applications, vol. 311, no. 2, pp. 495-505, 2005.

[17] H. A. H. Salem, "On the fractional order $m$-point boundary value problem in reflexive Banach spaces and weak topologies," Journal of Computational and Applied Mathematics, vol. 224, no. 2, pp. 565-572, 2009.

[18] L. S. Leibenson, "General problem of the movement of a compressible fluid in a porous medium," Izvestiia Akademii Nauk Kirgizskoi SSR, vol. 9, pp. 7-10, 1983.

[19] B. Ahmad, J. J. Nieto, A. Alsaedi, and M. El-Shahed, "A study of nonlinear Langevin equation involving two fractional orders in different intervals," Nonlinear Analysis: Real World Applications, vol. 13, no. 2, pp. 599-606, 2012.

[20] G. Dai, "Bifurcation and nodal solutions for $p$-Laplacian problems with non-asymptotic nonlinearity at 0 or $\infty$," Applied Mathematics Letters, vol. 26, no. 1, pp. 46-50, 2013.

[21] T. Chen, W. Liu, and C. Yang, "Antiperiodic solutions for Liénard-type differential equation with $p$-Laplacian operator," Boundary Value Problems, vol. 2010, Article ID 194824, 12 pages, 2010.

[22] D. Jiang and W. Gao, "Upper and lower solution method and a singular boundary value problem for the one-dimensional $p$ Laplacian," Journal of Mathematical Analysis and Applications, vol. 252, no. 2, pp. 631-648, 2000. 
[23] L. F. Lian and W. G. Ge, "The existence of solutions of $m$ point $p$-Laplacian boundary value problems at resonance," Acta Mathematicae Applicatae Sinica, vol. 28, no. 2, pp. 288-295, 2005.

[24] B. Liu and J. S. Yu, "Existence of solutions for the periodic boundary value problems with $p$-Laplacian operator," Journal of Systems Science and Mathematical Sciences, vol. 23, no. 1, pp. 76-85, 2003.

[25] J. J. Zhang, W. B. Liu, J. B. Ni, and T. Y. Chen, "Multiple periodic solutions of $p$-Laplacian equation with one-side Nagumo condition," Journal of the Korean Mathematical Society, vol. 45, no. 6, pp. 1549-1559, 2008.

[26] S. G. Samko, A. A. Kilbas, and O. I. Marichev, Fractional Integrals and Derivatives: Theory and Applications, Gordon and Breach Science Publishers, Amsterdam, The Netherlands, 1993.

[27] I. Podlubny, Fractional Differential Equations, vol. 198 of Mathematics in Science and Engineering, Academic Press, New York, NY, USA, 1999.

[28] A. A. Kilbas, H. M. Srivastava, and J. J. Trujillo, Theory and Applications of Fractional Differential Equations, Elsevier, Amsterdam, The Netherlands, 2006.

[29] C. F. Li, X. N. Luo, and Y. Zhou, "Existence of positive solutions of the boundary value problem for nonlinear fractional differential equations," Computers \& Mathematics with Applications, vol. 59, no. 3, pp. 1363-1375, 2010.

[30] M. A. Krasnosel'skii, Topological Methods in the Theory of Nonlinear Integral Equations, The Macmillan, New York, NY, USA, 1964. 


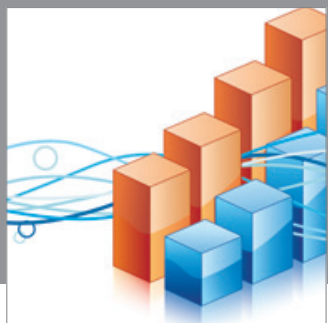

Advances in

Operations Research

mansans

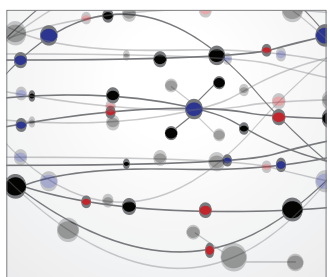

The Scientific World Journal
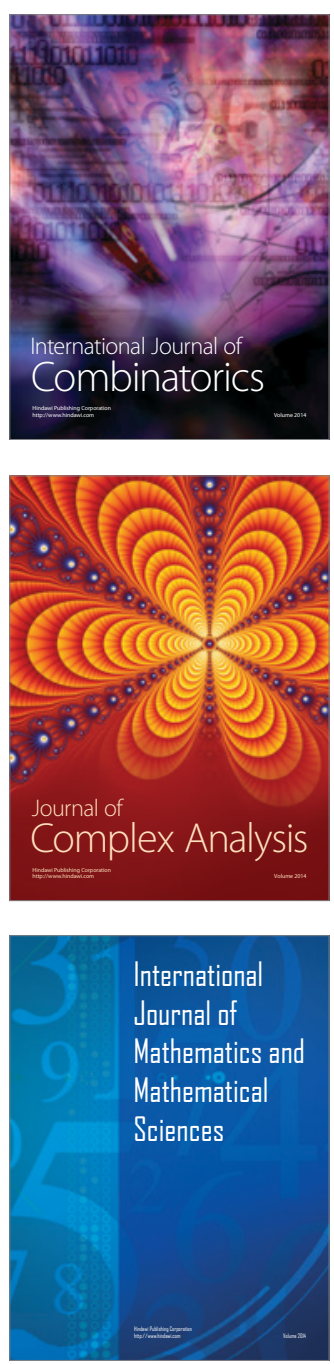
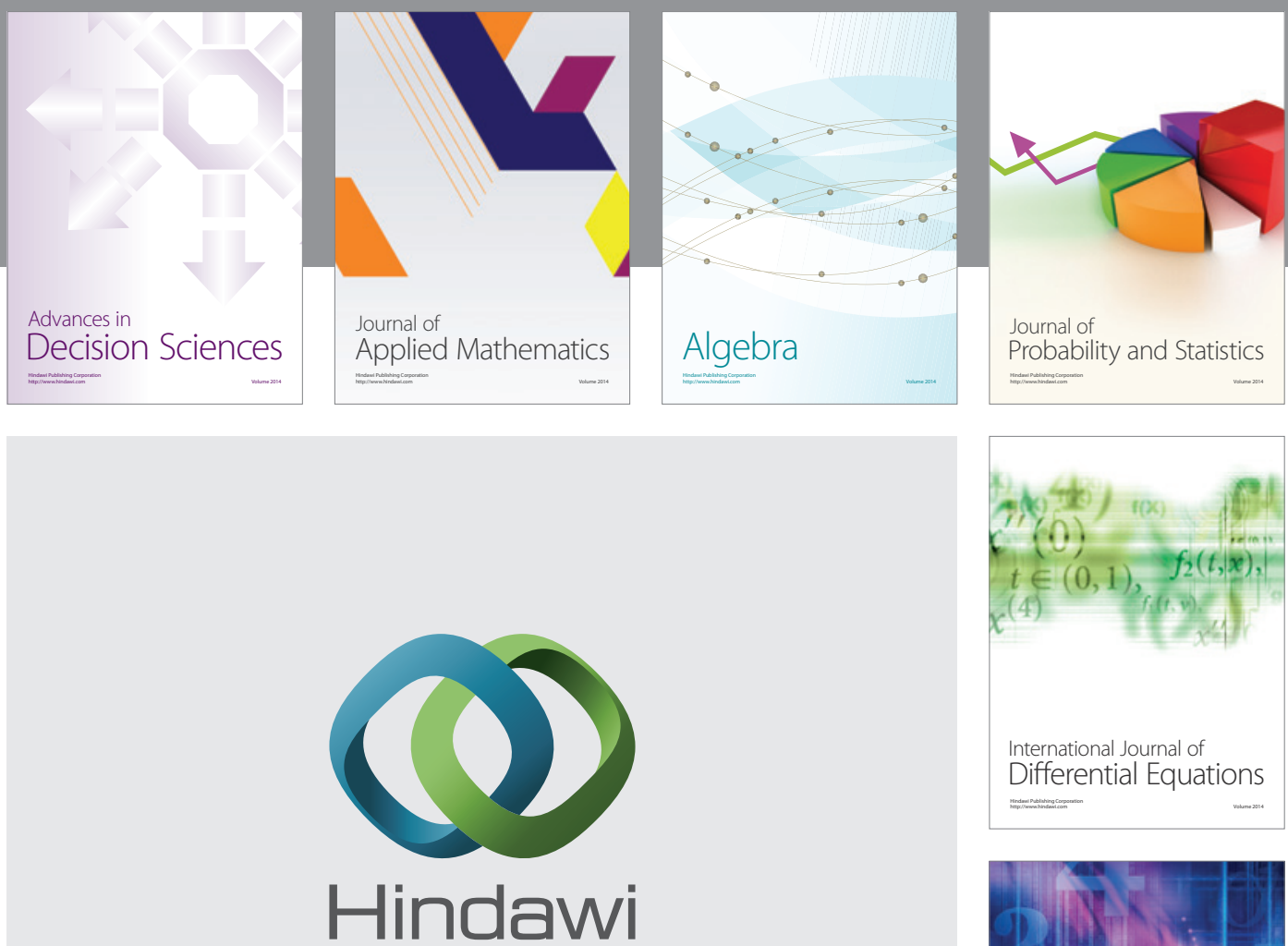

Submit your manuscripts at http://www.hindawi.com
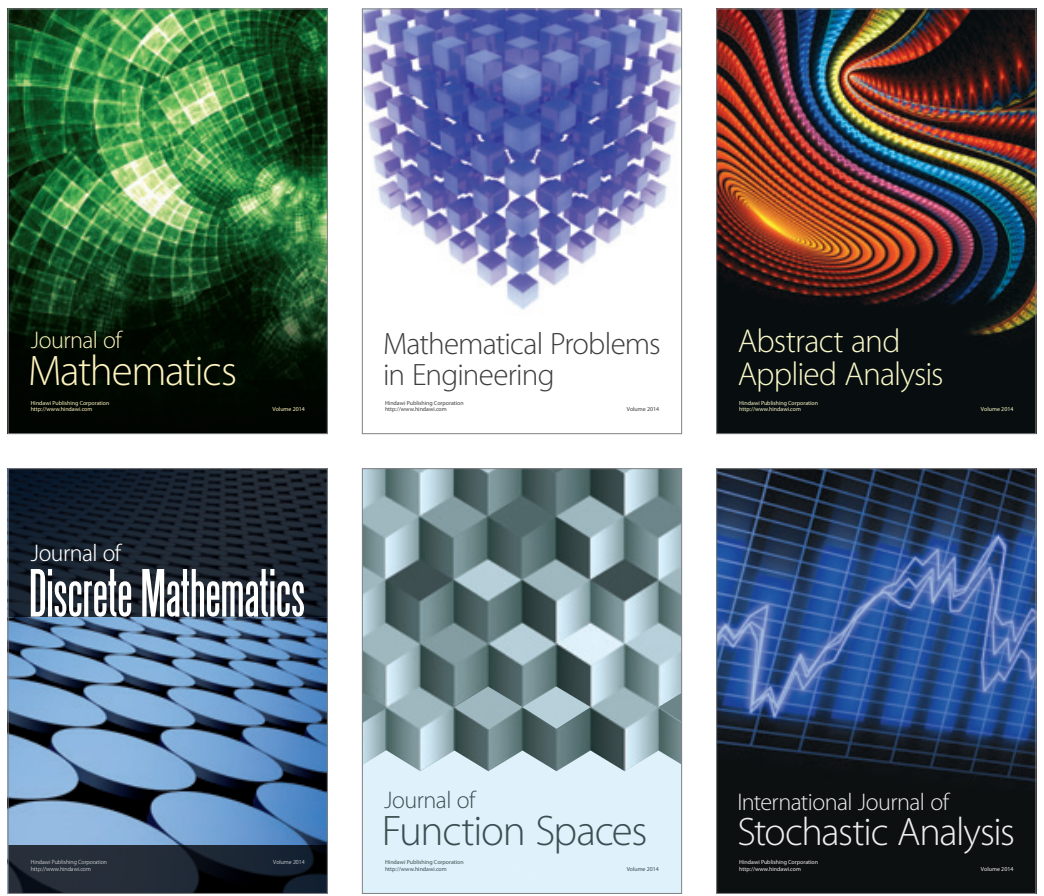

Journal of

Function Spaces

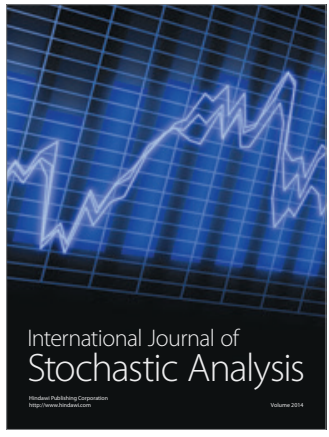

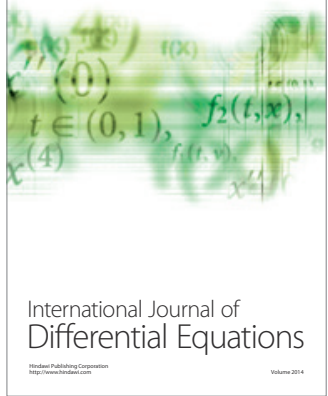
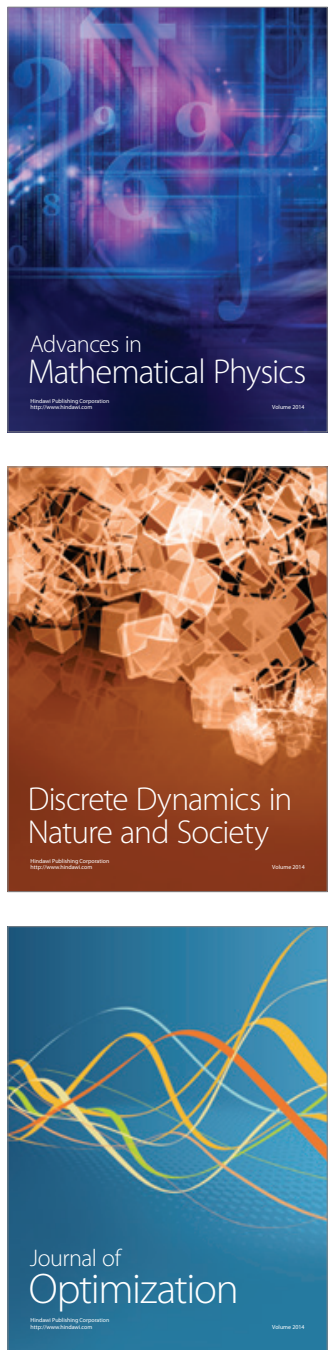